\title{
HOMOLOGY OPERATIONS ON INSTANTONS
}

\author{
CHARLES P. BOYER \& BENJAMIN M. MANN \\ To Margaret and Susie
}

In this paper we study the topology of certain moduli spaces of instantons which are of central importance in the Yang-Mills gauge theory in mathematical physics. These moduli spaces, which arise naturally in the differential geometric formulation of the Yang-Mills theory, have been extensively studied in recent years by many people including Atiyah [2], [3], Atiyah, Hitchin and Singer [6], Atiyah, Drinfeld, Hitchin and Manin [5], Atiyah and Bott [4], Atiyah and Ward [8], Donaldson [17], [18], [19], [20], Drinfeld and Manin [21], [22], [23], Taubes [48], [49], [50], [51], [52], and Uhlenbeck [54], [55], using various techniques from algebraic geometry, complex manifold theory, global analysis and twistor theory. In particular Donaldson's seminal work [17], [20] has shown that the moduli spaces of instantons contain remarkable geometric information.

This rich influx of ideas into topology from other areas of mathematics has inspired advances using more classical topological techniques, as exemplified by the work of Fintushel and Stern [25], [26], and it is natural to ask if homotopy theoretic techniques may also be profitably applied to study moduli spaces of instantons. Of particular interest in this regard is the foundational paper of Atiyah and Jones [7], which gave the first homological information about these moduli spaces over the four-sphere for arbitrary instanton number $k$ and formulated the basic topological questions in this subject. Specifically, Atiyah and Jones related these moduli spaces to components of well-known homotopy objects, namely configuration spaces and iterated loop spaces. In this paper we show that the disjoint union of these moduli spaces (where the union is taken over all positive instanton numbers $k$ ) behaves homologically like a four-fold iterated loop space (more precisely like a $C_{4}$ little cubes operad space in the sense of May [37]) with associated iterated loop space operations.

Received November 25, 1986 and, in revised form, July 30, 1987. The first author was partially supported by National Science Foundation grant DMS-8508950, and the second author by National Science Foundation grant DMS 8701539 and Clarkson University research grant 338-500. 
We then use these operations to obtain new information on the homology of these moduli spaces.

In $\S 1$ we briefly recall the differential geometric formulation of the YangMills gauge theory associated to a principal $G$-bundle $P_{k}$, where $G$ is a compact, connected, simple Lie group. We then define our main objects of study, $\mathscr{M}_{k}$, the moduli space of instantons with instanton number $k$, and $i_{k}: \mathscr{M}_{k} \rightarrow \mathscr{C}_{k}$, the natural inclusion of $\mathscr{M}_{k}$ into the moduli space of all connections on $P_{k}$. Technically we should index $\mathscr{M}_{k}$ by our choice of the Lie group $G$; however, as we are mainly concerned with the case $G=\operatorname{Sp}(n)$ in the first six sections of this paper, and we restrict out attention to the case $G=\operatorname{Sp}(1)=S^{3}$ in $\S \S 7-10$, we have chosen to keep the notation as simple as possible.

$\S 2$ briefly reviews what is known about the topology of $\mathscr{M}_{k}$, highlighting the work of Atiyah and Jones [7]. We state the topological questions that arise naturally from their work, including the Atiyah-Jones conjecture on the low dimensional homotopy of $\mathscr{M}_{k}$. We then see precisely how the disjoint union of the $\mathscr{M}_{k}$, over $k>0$, is surrounded by four-fold loop spaces. This, in turn, suggests that the moduli spaces $\mathscr{M}_{k}$ have a rich homological structure.

$\S 3$ is a technical section which recalls some basic facts from May's theory [36] of iterated loop spaces. More precisely, we recall the definition of $C_{4}$ operads and $C_{4}$ operad spaces. These objects are the proper generalization of four-fold loop spaces needed to enrich the homotopy structure of $\amalg \mathscr{M}_{k}=\mathscr{M}$ in a homotopically compatible way with known iterated loop structures on iterated loop spaces. We then observe that the existence of such a $C_{4}$ structure on $\amalg \mathscr{M}_{k}$ is not immediate and in the succeeding three sections we construct our desired homotopy structure.

In $\S 4$ we give Atiyah's geometric formulation [2] of the Atiyah, Drinfeld, Hitchin and Manin [5] construction of the entire moduli space $\mathscr{M}_{k}$ in terms of linear algebra. This description and a result of Taubes [50] given in $\S 2$ are the crucial ingredients used in the construction of our $C_{4}$ little cubes maps on $\amalg \mathscr{M}_{k}=\mathscr{M}$ given $\S \S 5$ and 6 .

$\S 5$ contains our key technical result. Here we prove that the moduli spaces admit loop sum maps $*: \mathscr{M}_{k} \times \mathscr{M}_{l} \rightarrow \mathscr{M}_{k+l}$ that agree, up to homotopy, with the standard loop sum map on certain four-fold loop spaces (see Theorem 5.2). In $\S 6$ we extend this result from the loop sum maps to $C_{4}$ operad maps on $\amalg \mathscr{M}_{k}$ that agree, up to homotopy, with the standard $C_{4}$ operad structure on these same four-fold loop spaces (see Theorem 6.10). These facts permit the homology calculations which occupy the rest of the paper. 
In the next four sections we restrict our attention to the case $G=\operatorname{Sp}(1)$. $\S 7$ analyzes the case when the instanton number is 1 and identifies the natural inclusion $\mathscr{M}_{1} \rightarrow \mathscr{C}_{1}$ with the classical $J$-homomorphism. Although this appears to be a rather trivial case it has far-reaching consequences as seen in the computations given in $\S \S 9$ and 10 .

In $\S 8$ we briefly review the theory of homology operations on iterated loop spaces (and $C_{4}$ operad spaces). The results of previous sections imply we may apply this theory to $\amalg \mathscr{M}_{k}$ and obtain information on $H_{*}\left(\mathscr{M}_{k}, Z / p\right)$ for $p$ a prime number. We then catalog our results for $p=2$ in $\S 9$ and for $p$ an odd prime in $\S 10$.

$\S 11$ concludes with a list of open questions raised by our computations and suggests directions for further study. The techniques developed in this paper should prove useful in studying moduli spaces of instantons associated to other Lie groups, moduli spaces of instantons associated to bundles over more general compact smooth four-manifolds, and moduli spaces for stable holomorphic vector bundles. Finally, we have enclosed an appendix with explicit calculations for $H_{q}\left(\mathscr{M}_{k}, Z / p\right)$ for some small values of $k$.

We would like to thank Fred Cohen, Jacques Hurtubise, John Jones, Jim Milgram and Cliff Taubes for valuable comments, observations and suggestions which have greatly helped us during the preparation of this paper.

\section{Yang-Mills instantons}

We begin this paper with a very brief review of the differential geometric formulation of the Yang-Mills theory on principal bundles. We give sufficient details to make this paper reasonably self-contained and to establish basic notation used throughout the paper. The reader unfamiliar with the foundational papers in this area ([2], [5], [6], [7], [8], [21], and [40]) is encouraged to consult these sources.

Let $G$ be a compact, connected, simple Lie group (we are interested mainly in the compact simple classical groups) and let $\pi: P \rightarrow S^{4}$ be a principal $G$ bundle over the four-sphere. Recall that such bundles are indexed by the integers and we write $P_{k}$ for the bundle classified by the map $S^{4} \rightarrow B G$ of degree $k$ (recall $\pi_{3}(G)=Z$ ). There are two natural geometric spaces associated to $P_{k}$, the space of all connections, $\mathscr{A}_{k}$, and the gauge group, $\mathscr{G}\left(P_{k}\right) . \mathscr{A}_{k}$ is well known to be an affine space [32], and the gauge group is defined as follows:

Definition 1.1. $\mathscr{G}\left(P_{k}\right)=\left\{f \mid f: P_{k} \rightarrow P_{k}\right.$ is a bundle automorphism which covers the identity map on $\left.S^{4}\right\}$. 
Definition 1.2. $\mathscr{G}^{b}\left(P_{k}\right)$, the based gauge group of $P_{k}$, is the normal subgroup of $\mathscr{G}\left(P_{k}\right)$ given by all $f \in \mathscr{G}\left(P_{k}\right)$ such that $f$ is the identity map on the fiber over a distinguished base point.

If we think of $\omega \in \mathscr{A}_{k}$ as a pseudotensorial 1-form, then $\mathscr{G}\left(P_{k}\right)$, and thus $\mathscr{G}^{b}\left(P_{k}\right)$, act on $\mathscr{A}_{k}$ via the pullback; that is,

$$
\omega \mapsto f^{*}(\omega)=\operatorname{ad}_{f^{-1}} \omega+f^{-1} d f .
$$

Here we have identified $\mathscr{G}\left(P_{k}\right)$ with ad-equivariant maps $f: P_{k} \rightarrow G$ [27]. While the action of all of $\mathscr{G}\left(P_{k}\right)$ on $\mathscr{A}_{k}$ is not free, this action restricted to the based gauge group $\mathscr{G}^{b}\left(P_{k}\right)$ is free. This observation and a local slice analysis [7], [45], show

Proposition 1.4. $\mathscr{A}_{k} / \mathscr{G}^{b}\left(P_{k}\right)=\mathscr{C}_{k}$ is a principal $\mathscr{G}^{b}\left(P_{k}\right)$ bundle where $\mathscr{C}_{k}$ may be identified with $B \mathscr{G}^{b}\left(P_{k}\right)$, the classifying space of $\mathscr{G}^{b}\left(P_{k}\right)$. Furthermore, $\mathscr{C}_{k}$ is homotopy equivalent to $\Omega_{k}^{3} G \simeq \Omega_{k}^{4} B G$.

Here $\Omega X$ is the space of based loops on $X$; that is, the space of based maps $f:\left(S^{1}, 1\right) \rightarrow(X, *)$ with the compact open topology. Recall $\Omega X$ is an $H$-space with operation (classically called the loop sum)

$$
f * g\left(e^{i \vartheta}\right)= \begin{cases}f\left(e^{2 i \vartheta}\right) & \text { if } 0 \leq \vartheta \leq \pi \\ g\left(e^{2 i \vartheta}\right) & \text { if } \pi \leq \vartheta \leq 2 \pi\end{cases}
$$

We may iterate this construction to obtain $\Omega^{n}(X)=\Omega(\Omega \cdots(\Omega X))$, the space of $n$-fold iterated loops on $X$; equivalently, $\Omega^{n}(X)$ is homeomorphic to the space of based maps $f:\left(S^{n}, 1\right) \rightarrow(X, *)$. As $\pi_{3}(G)=Z$ the components of $\Omega^{3} G$ are indexed by the integers and we write $\Omega_{k}^{3} G$ for the component of $\Omega^{3} G$ consisting of all based maps $f:\left(S^{3}, 1\right) \rightarrow(G$, id $)$ of degree $k$. The fact that $\mathscr{C}_{k}$ may be identified, up to homotopy, with a four-fold loop space has a pervasive influence throughout this paper.

Given a representation $\rho: G \rightarrow$ Aut $V$ of $G$ on a vector space $V$ we may form the associated vector bundle $E_{k}=P_{k} \times_{G} V$ [32]. Of course connections in $P_{k}$ give rise to connections in $E_{k}$ and vice versa. Now let $\omega \in \mathscr{A}_{k}$. Its curvature $F^{\omega}=D^{\omega} \omega$ is a section of the vector bundle $\left(P_{k} \times_{G} g\right) \otimes \Lambda^{2}\left(S^{4}\right)$ where $g$ is the Lie algebra of $G$ and $\rho: G \rightarrow \operatorname{Aut}(g)$ is the adjoint representation. There is a natural bilinear form on $\left(P_{k} \times_{G} g\right) \otimes \Lambda^{2}\left(S^{4}\right)$ given by the Hodge inner product on $\Lambda\left(S^{4}\right)$ (with respect to the standard metric on $S^{4}$ ) and the Killing form on $g$. The corresponding norm gives the Yang-Mills functional on $\mathscr{A}_{k}$ :

$$
\mathscr{Y} \mathscr{M}(\omega)=\int_{S^{4}}\left\|F^{\omega}\right\|^{2} .
$$

Furthermore, $F^{\omega}$ splits orthogonally into self-dual, $F_{+}^{\omega}$, and anti-self-dual, $F_{-}^{\omega}$, components (with respect to the Hodge decomposition) and we may 
rewrite the integral as

$$
\mathscr{Y} \mathscr{M}(\omega)=\int_{S^{4}}\left\|F_{+}^{\omega}\right\|^{2}+\left\|F_{-}^{\omega}\right\|^{2}
$$

Now Chern-Weil theory [32] yields

$$
p_{1}(g)=\frac{1}{4 \pi^{2}} \int_{S^{4}}\left\|F_{+}^{\omega}\right\|^{2}-\left\|F_{-}^{\omega}\right\|^{2}
$$

where $p_{1}(g)$ is the first Pontrjagin number of the adjoint bundle $P_{k} \times_{G} g$. Thus the self-dual, $F^{\omega}=F_{+}^{\omega}$ (for $k>0$ ), and the anti-self-dual, $F^{\omega}=F_{-}^{\omega}$ (for $k<0$ ), connections give the absolute minima of $\mathscr{Y} \mathscr{M}$. These absolute minima are called instantons and anti-instantons respectively. As any orientation reversing diffeomorphism will pull back $P_{k}$ to $P_{-k}$ and pull back instantons to anti-instantons (or anti-instantons to instantons depending on the sign of $k$ ) it suffices to restrict our attention to $k>0$ and instantons. We do so for the remainder of the paper.

Let $\mathscr{I}_{k} \subset \mathscr{A}_{k}$ denote the subspace of instanton (self-dual) connections in $\mathscr{A}_{k}$. Further we let $\hat{\mathscr{A}}_{k} \subset \mathscr{A}_{k}$ denote the subspace of all irreducible connections on $P_{k}$ and set $\hat{\mathscr{I}}_{k}=\mathscr{J}_{k} \cap \mathscr{A}_{k}$. For $G=\mathrm{SU}(2)=\mathrm{Sp}(1)$ and $k>0$ it follows from the fact that there are no harmonic 2-forms on $S^{4}$ that $\mathscr{A}_{k}=\hat{\mathscr{A}}_{k}$ and thus $\mathscr{I}_{k}=\hat{\mathscr{I}}_{k}$ but these equalities are definitely false for all other compact simple Lie groups.

Most importantly a direct calculation shows that the Yang-Mills functional, $\mathscr{Y} \mathscr{M}$, is invariant under the action of the gauge group $\mathscr{G}\left(P_{k}\right)$ on $\mathscr{A}_{k}$ given in (1.3). Thus we obtain the following moduli spaces which are our fundamental objects of interest.

Definition 1.9. $\mathscr{M}_{k}=\mathscr{J}_{k} / \mathscr{G}^{b}\left(P_{k}\right)$ is the based moduli space of all instantons.

Definition 1.10. $\mathscr{M}_{k}^{\prime}=\mathscr{I}_{k} / \mathscr{G}\left(P_{k}\right)$ is the moduli space of all instantons.

Definition 1.11. $\hat{\mathscr{M}}_{k}=\hat{\mathscr{I}}_{k} / \mathscr{G}^{b}\left(P_{k}\right)$ is the based moduli space of all irreducible instantons.

Definition 1.12. $\quad \hat{\mathscr{M}}_{k}^{\prime}=\hat{\mathscr{I}}_{k} / \mathscr{G}\left(P_{k}\right)$ is the moduli space of all irreducible instantons.

A theorem of Atiyah, Hitchin and Singer [6] shows that $\hat{\mathscr{M}}_{k}^{\prime}$ is either empty or is a smooth manifold of dimension $p_{1}(g)-\operatorname{dim}(g)$. Furthermore the factor group $\mathscr{G}\left(P_{k}\right) / \mathscr{G}^{b}\left(P_{k}\right)$ is naturally identified with $G / Z$ and by $(1.3)$ we have a principal bundle

$$
G / Z \rightarrow \hat{\mathscr{M}}_{k} \rightarrow \hat{\mathscr{M}}_{k}^{\prime}
$$


where $\hat{\mathscr{M}}_{k}$ is a smooth manifold of dimensions $p_{1}(g)$. Here $Z$ is the center of $G$. We also have the obvious commutative diagram:

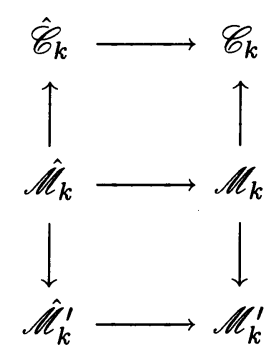

While $\mathscr{M}_{k}$ is a smooth manifold for any Lie group $G$ (cf. [49]), if $G \neq$ $\mathrm{Sp}(1)$ then $\mathscr{M}_{k}^{\prime}$ is not a smooth manifold and $\mathscr{M}_{k} \rightarrow \mathscr{M}_{k}^{\prime}$ is not a principal $G / Z$ bundle. However these spaces still have a rather interesting piece of topological structure and it is desirable to study all spaces and maps occurring in diagram (1.14). For any compact simple $G, p_{1}(g)$ can be computed in terms of the degree $k$ and the rank of $G$ (see [6]). In fact $p_{1}(g)=a(g) k$ where the proportionality constant depends only on $g$. However we will be mainly interested in the case $G=\operatorname{Sp}(n)$ where $a(g)=4(n+1)$ so $p_{1}(g)=4(n+1) k$, thus $\operatorname{dim} \hat{\mathscr{M}}_{k}=4(n+1) k$, and $\operatorname{dim} \hat{\mathscr{M}}_{k}^{\prime}=4(n+1) k-n(2 n+1)$. If there exist irreducible self-dual connections on $\operatorname{Sp}(n) \rightarrow P_{k} \rightarrow S^{4}$ then $k \geq n$ [6]. Of special interest is the case $G=\operatorname{Sp}(1)$ for then $\hat{\mathscr{M}}_{k}=\mathscr{M}_{k}$ and $\hat{\mathscr{M}}_{k}^{\prime}=\mathscr{M}_{k}^{\prime}$ are nonempty smooth manifolds of dimension $8 k$ and $8 k-3$ respectively for all $k>0$.

We conclude our review of instantons with the well-known construction of a subclass of $\mathrm{Sp}(1)$ instantons due to 't Hooft [53], which come from configurations of $k$ unordered points in $R^{4}$. Recall the set of $k$ distinct points in $R^{4}$ is an open submanifold of $R^{4}$ which admits an obvious free action of the symmetric group $\Sigma_{k}$. The quotient space $C_{k}\left(R^{4}\right)$ is called the configuration space of $k$ points in $R^{4}$ and has played a key role in the homology theory of iterated loop spaces (see, for example, [11], [16], [24], [38], [39], [43]). Viewing $R^{4}$ as the quaternionic plane $H^{1}$ and $S^{4}$ as the quaternionic projective plane $H P(1)$ we may construct an instanton as follows: Choose $k$ distinct points $\left(a_{1}, a_{2}, \cdots, a_{k}\right)$ in $H^{1}$ and define a map $f: H P(1) \rightarrow H P(k)$ by the formula $f(x)=\left(1,\left(x-a_{1}\right)^{-1}, \cdots,\left(x-a_{k}\right)^{-1}\right)$ where $\left(x-a_{i}\right)^{-1}$ is $\infty$ if $x=a_{i}$. Fixing a standard connection $\omega$ on the Hopf fibration $S^{3} \rightarrow S^{4 k+3} \rightarrow H P(k)$ we obtain a self-dual connection $f^{*}(\omega)$ on $P_{k} \rightarrow S^{4}=H P(1)$. This assignment gives rise to the first inclusion $j_{k}$ in

$$
C_{k}\left(R^{4}\right) \stackrel{j_{k}}{\longrightarrow} \mathscr{M}_{k} \stackrel{i_{k}}{\longrightarrow} \mathscr{C}_{k}
$$


Atiyah and Jones [7] first noticed (1.15) and in the next section we review their work relating the topology of $\mathscr{M}_{k}$ to the topology of the better known spaces $C_{k}\left(R^{4}\right)$ and $\mathscr{C}_{k} \simeq \Omega_{k}^{3} S^{3}$.

\section{On the topology of instantons}

In this section we review the main result of Atiyah and Jones [7] on the homology of the inclusion maps $i_{k}: \mathscr{M}_{k} \rightarrow \mathscr{C}_{k}$, recall the questions they pose that arise from their analysis and summarize what is known about $\pi_{*}\left(\mathscr{M}_{k}\right)$, $H_{*}\left(\mathscr{M}_{k}\right)$ and the induced maps $i_{k^{*}}$. We are then able to describe the main program of this paper and conclude with a theorem of Taubes which plays a key technical role in our analysis.

We begin with $G=\operatorname{Sp}(1)$ and start with the inclusions $C_{k} \stackrel{j_{k}}{\longrightarrow} \mathscr{M}_{k} \stackrel{i_{k}}{\longrightarrow} \mathscr{C}_{k}$ described at the end of $\S 1$. Segal [43] and Boardman and Vogt [11] have shown there are maps $S_{k}: C_{k}\left(R^{n}\right) \rightarrow \Omega_{k}^{n} S^{n}$ which, for all $k$ and $n$, are homology (although not homotopy) equivalences through a range (that depend on $k$ and $n$ ). Furthermore Atiyah and Jones showed the following diagram commutes for all $k$ :

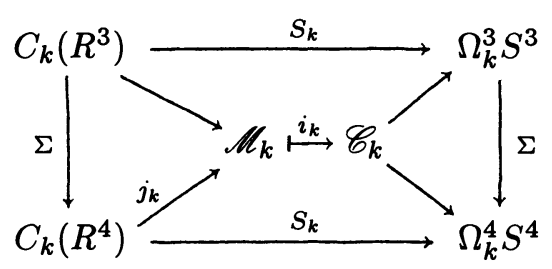

where $\Sigma$ represent the standard suspension maps. Using this commutative diagram and the Segal, Boardman-Vogt result, Atiyah and Jones obtained the following.

Theorem 2.2 [7]. Let $G=\operatorname{Sp}(1)$. Then $\left(i_{k}\right)_{q}: H_{q}\left(\mathscr{M}_{k}\right) \rightarrow H_{q}\left(\mathscr{C}_{k}\right)$ is a surjection for $q \ll k$.

Atiyah and Jones then pose the following questions:

1. Is $\left(i_{k}\right)_{q}$ actually a homology isomorphism through a range?

2. Can the range of the surjection (isomorphism) $q=q(k)$ be explicitly determined as a function of $k$ ?

3. Is $\left(i_{k}\right)_{q}$ a surjection on homotopy groups through a range? Even better is $i_{k}$ actually a homotopy equivalence through a range?

4. Are similar results true if $\operatorname{Sp}(1)$ is replaced by more general compact simple $G$ ? Notice that immediately there arises a new complication as $\hat{\mathscr{M}}_{k}$ is properly contained in $\mathscr{M}_{k}$ for all $G \neq \mathrm{Sp}(1)$. In fact $\hat{\mathscr{M}}_{k}$ may be empty while at the same time $\mathscr{M}_{k}$ may be quite large. Thus this question really asks one 
to analyze the commutative diagrams in homology and homotopy induced by diagram (1.14).

Question 3, which asks if $i_{k}: \mathscr{M}_{k} \rightarrow \mathscr{C}_{k}$ is a homotopy equivalence through a range and is now commonly known as the Atiyah-Jones conjecture, still remains open at this time. It might be helpful at this point to review precisely what is known about the topology of $\mathscr{M}_{k}$. Again let $G=\operatorname{Sp}(1)$.

Theorem 2.3 (Donaldson [18]). $\mathscr{M}_{k}$ is a complex manifold.

Theorem 2.4 (Taubes [50]). $\pi_{0}\left(\mathscr{M}_{k}\right)=1$ for all $k$.

Theorem 2.5 (Hartshorne [28]). $\quad \pi_{1}\left(\mathscr{M}_{2}\right)=Z / 2$.

Theorem 2.6 (Hurtubise [30]). $\quad \pi_{1}\left(\mathscr{M}_{k}\right)=Z / 2$ for all $k>1$.

Furthermore, Taubes, using techniques from partial differential equations, global analysis and infinite-dimensional Morse theory, Kirwan, using techniques from symplectic geometry, and Segal, using techniques from analytic loop groups, have all initiated powerful attacks on the Atiyah-Jones conjecture. In fact, Taubes [52] now has both a proof of a stable version of the Atiyah-Jones conjecture as well as a strong generalization of Theorem 2.2 for arbitrary Lie groups. We are not in a position to properly explain their work here and now leave that subject to concentrate on results in homology.

As the homology of $C_{k}\left(R^{n}\right)$ and $\Omega_{k}^{n} S^{n}$ is well known ([16], [24], [38], and [43]) it is possible to use diagram (2.1) to construct nonzero classes in the image $\left(i_{k}\right)_{q}: H_{q}\left(\mathscr{M}_{k}\right) \rightarrow H_{q}\left(\mathscr{C}_{k}\right)$. We will see in $\S 9$ that if one works with homology with $Z / 2$ coefficients then this method yields nonzero classes for $q \leq 2^{r+1}-2$ when $k=2^{r}$. However it is not possible to directly use this method to obtain interesting classes in $H_{q}\left(\mathscr{M}_{k}\right)$ when $q>k / 4$.

For reasons that will quickly become apparent it is natural to take the union, over all positive values of $k$, of diagram (2.1) to obtain the following commutative diagram:

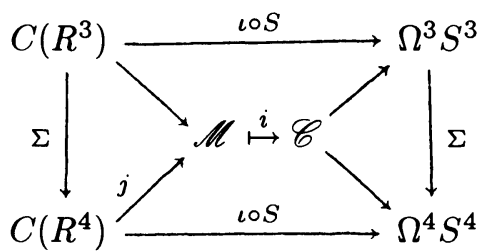

where $C\left(R^{n}\right)=\bigsqcup_{k>0} C_{k}\left(R^{n}\right), \mathscr{M}=\bigsqcup_{k>0} \mathscr{M}_{k}, \mathscr{C}=\bigsqcup_{k>0} \mathscr{C}_{k}$, and $\iota$ is the natural inclusion of the positively indexed path components into the total iterated loop space.

The inclusions $\iota \circ S$ are well known to be group completions ([43], [11]), and thus we find that $\amalg \mathscr{M}_{k}=\mathscr{M}$ is completely surrounded by iterated loop spaces (or spaces that group complete to iterated loop spaces). The homology theory of such spaces is very rich (see $\S \S 3$ and 8 for a brief review) and it is clear that 
if one could enrich $\mathscr{M}$ with such an "iterated loop space structure" so that diagram (2.7) respects the additional structure (at least up to homotopy) then it would be possible to learn much more about $H_{*}(\mathscr{M})$ and $H_{*}\left(\mathscr{M}_{k}\right)$. This entire paper consists of carrying out this program. For example Corollaries 9.9 and 10.9 will give nonzero classes in $H_{6 k-3}\left(\mathscr{M}_{k}\right)$ for infinitely many $k$.

If we replace $G=\operatorname{Sp}(1)$ by $G=\operatorname{Sp}(n)$ we may still map the configuration spaces $C_{k}\left(R^{4}\right)$ into $\mathscr{M}_{k}$ (see $\S 4$ and the remarks after Proposition 4.16) and diagram (2.7) can be replaced by the following analogous commutative diagram:

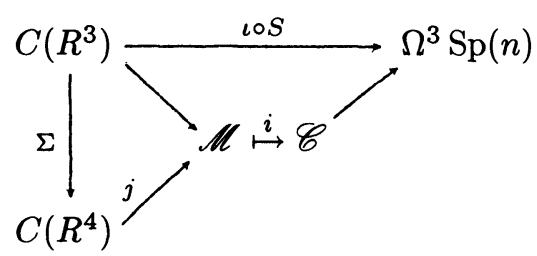

The only essential difference between diagrams (2.7) and (2.8) is that the inclusion of the configuration space $\amalg C_{k}\left(R^{4}\right)$ into $\Omega^{3} \mathrm{Sp}(n) \simeq \Omega^{4} B \operatorname{Sp}(n)$ is no longer a group completion. Fortunately this distinction is irrelevant for our purposes and all the structures we wish to study are preserved in (2.8). Thus we are able to give a unified treatment in describing the additional homotopy structure on $\mathscr{M}$ for all $\operatorname{Sp}(n)$ in the following four sections. Only when we turn to specific homological computations (in $\S \S 7-10$ of the paper) will we restrict our attention to the case $n=1$ again.

In order to carry out our program we will require the following theorem of Taubes [50], which was an ingredient in his proof that $\pi_{0}\left(\mathscr{M}_{k}\right)=1$ for $G=\mathrm{SU}(2)$ (and $\mathrm{SU}(3)$ ). We note that $G$ is not restricted to $\mathrm{SU}(2)$ or $\mathrm{SU}(3)$ in the following

Theorem 2.9 (Taubes [50]). Let $P_{k} \rightarrow S^{4}$ be a principal $G$-bundle with $k \geq 0$. Then there exist $\varepsilon>0$ and a strong deformation retract $\mathscr{C}_{k}^{\varepsilon}$ onto $\mathscr{M}_{k}$. Here $\mathscr{C}_{k}^{\varepsilon}=\mathscr{Y} \mathscr{M}^{-1}([0, \varepsilon))$ where we have normalized $\mathscr{Y} \mathscr{M}: \mathscr{C}_{k} \rightarrow R$ as in [50] so that $\mathscr{Y} \mathscr{M}\left(\mathscr{M}_{k}\right)=0$.

We shall use Theorem 2.9 in a crucial way in $\S \S 5$ and 6 to enrich $\mathscr{M}$ with additional homological structure.

\section{Iterated loop spaces}

We have just seen that $\mathscr{M}=\amalg \mathscr{M}_{k}$ appears to be intimately related to the four-fold iterated loop space $\Omega^{4} B \operatorname{Sp}(n)$. Thus it seems appropriate at this point to recall some basic facts about the theory of iterated loop spaces and the machinery which we will need to tie $\mathscr{M}$ more precisely into this theory. 
Actually we expand our attention from iterated loop spaces to May's $C_{n}$ operad spaces [36], which are based on Boardman and Vogt's little $n$-cube spaces [11]. This is the proper generalization of iterated loop spaces needed to study the spaces and maps occurring in our fundamental commutative diagram (2.8) and gives the largest class of spaces on which the homology operations we study later are defined. In $\S \S 5$ and 6 we will show that, up to homotopy, $\mathscr{M}$ behaves like a $C_{4}$ space and, that up to homotopy, diagram (2.8) behaves like a commutative diagram of $C_{4}$ spaces (with compatible $C_{4}$ structures). Thus we now briefly summarize facts used in later sections to establish a "homotopy $C_{4}$ " structure on $\mathscr{M}$ and to compute homology operations in $H_{*}(\mathscr{M})$.

Definition $3.1([11],[36])$. Let $I^{n}$ be the unit $n$-cube and let $J^{n}=\dot{I}^{n}$ be the interior of $I^{n}$. An open little $n$-cube is an affine embedding $f$ of $J^{n}$ into $J^{n}$ with parallel axes. Then $C_{n}(j)$ is the space of $j$-tuples $\left(f_{1}, \cdots, f_{j}\right)$ of open little $n$-cubes with disjoint images in $J^{n} \subset I^{n}$ (with the subspace topology inherited from $\left.\operatorname{Map}\left(\Pi^{j} J^{n}, J^{n}\right)\right)$.

Notice that the symmetric group $\Sigma_{j}$ acts on $C_{n}(j)$ by permuting the disjoint images of the little $n$-cubes. Now $C_{n}(j)$ acts on $\Omega^{n} X$ in the following way. First we identify $\Omega^{n} X$ with the space of maps $\left(I^{n}, \partial I^{n}\right) \rightarrow(X, *)$. Then

$$
\vartheta_{n, j}: C_{n}(j) \times_{\Sigma_{j}}\left(\Omega^{n} X\right)^{j} \rightarrow \Omega^{n} X
$$

is defined by mapping the image of the $i$ th little $n$-cube in $J^{n} \subset I^{n}$ via the $i$ th coordinate function of $\left(\Omega^{n} X\right)^{j}$ into $X$ and mapping the complement of the images of all $j$ little $n$-cubes in $I^{n}$ to the base point $* \in X$. If $\Sigma_{j}$ acts on $\left(\Omega^{n} X\right)^{j}$ by permuting the $j$ coordinates then it is clear that $\vartheta_{n, j}$ is well defined.

These structures satisfy many other compatibility conditions, for example, the following diagram (see May [36]) is known to commute.

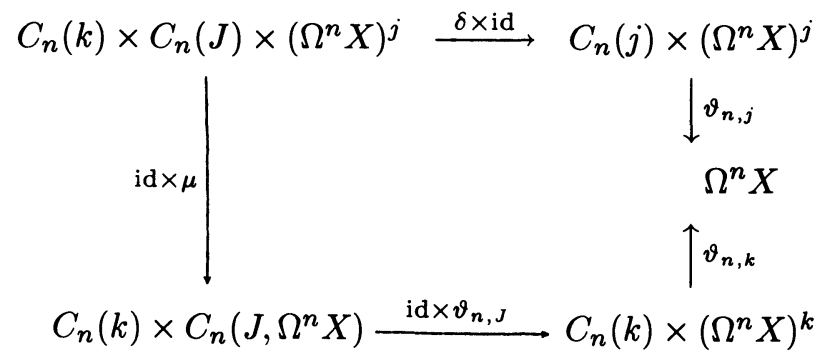

Here $J=\left(j_{1}, \cdots, j_{k}\right), \sum_{l=1}^{k} j_{l}=j, C_{n}(J)=C_{n}\left(j_{1}\right) \times \cdots \times C_{n}\left(j_{k}\right)$, $C_{n}\left(J, \Omega^{n} X\right)=C_{n}\left(j_{1}\right) \times\left(\Omega^{n} X\right)^{j_{1}} \times \cdots \times C_{n}\left(j_{k}\right) \times\left(\Omega^{n} X\right)^{j_{k}}, \mu$ is the shuffle homeomorphism, and $\delta: C_{n}(k) \times C_{n}\left(j_{1}\right) \times \cdots \times C_{n}\left(j_{k}\right) \rightarrow C_{n}(j)$ is defined by $\delta\left(g ; f_{1}, \cdots, f_{k}\right)=g\left(f_{1}+\cdots+f_{k}\right)$, where + denotes disjoint union. 
Thus $\delta$ places the $j_{i}$ disjoint little $n$-cubes of $f_{i} \in C_{n}\left(j_{i}\right)$ homeomorphically into the interior of the $i$ th little $n$-cube of $g \in C_{n}(k)$. Also note (3.3) is equivariant with respect to the obvious actions of the various symmetric groups $\Sigma_{k}, \Sigma_{j}, \Sigma_{j i}$

These structure maps are formalized in both Boardman and Vogt's [11] and May's [36] theories of iterated loop spaces. More precisely, $C_{n}$, the union over all $j \geq 0$ of the $C_{n}(j)$ 's together with the structure maps $\delta: C_{n}(k) \times C_{n}\left(j_{1}\right) \times$ $\cdots \times C_{n}\left(j_{k}\right) \rightarrow C_{n}(j)$, is an operad (see [36, Definition 1.1 and Theorem 4.1]). Furthermore the totality of the structure maps $\left\{\vartheta_{n, j}\right\}$ together with higher compatibilities such as (3.3) make $\Omega^{n} X$ a $C_{n}$ space (see [36, Definition 1.2, Lemma 1.4 and Theorem 5.1]). We now need the following key definition of May.

Definition 3.4 [36]. $\quad Y$ is a $C_{n}$ space if it is equipped with structure maps $\vartheta_{j}: C_{n}(j) \times Y^{j} \mapsto Y$ for all $j \geq 0$ such that

(a) The following (the analogue of diagram (3.3)) commutes:

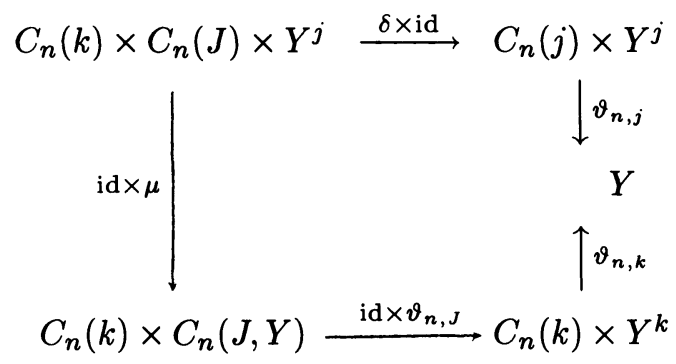

(b) $\vartheta_{1}(1, y)=y$ for all $y \in Y$.

(c) $\vartheta_{j}(c \sigma, z)=\vartheta_{j}(c, \sigma z)$ for $c \in C_{n}(j), z \in Y^{j}$ and $\sigma \in \Sigma^{j}$.

Furthermore May defines a $C_{n}$ map between $C_{n}$ spaces to be a map $f:(Y, \vartheta)$ $\rightarrow\left(Y^{\prime}, \vartheta^{\prime}\right)$ such that the following diagrams commute for all $j$ :

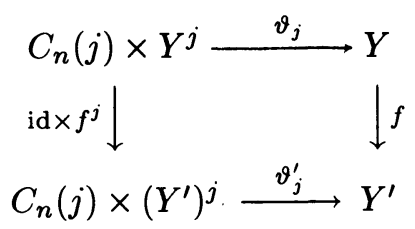

Returning to diagram (2.8) we find the composition of inclusions

$$
\coprod C_{k}\left(R^{4}\right) \rightarrow \mathscr{M} \rightarrow \mathscr{C} \rightarrow \Omega^{4} B \operatorname{Sp}(n),
$$

where every space but $\mathscr{M}$ is known to be a $C_{4}$ space and the total composite map is known to be a $C_{4}$ map.

Notice however any naive attempt to impose a compatible $C_{4}$ structure on $\mathscr{M}$ by directly minimizing the little cubes action on $\Omega^{4} B \operatorname{Sp}(n)$ fails. This is 
evident even at the loop sum level in trying to construct a map $\mathscr{M}_{k} \times \mathscr{M}_{l} \stackrel{*}{\rightarrow}$ $\mathscr{M}_{k+l}$ that commutes with the map $\Omega_{k}^{4} B \operatorname{Sp}(n) \times \Omega_{l}^{4} B \operatorname{Sp}(n) \stackrel{*}{\rightarrow} \Omega_{k+l}^{4} B \operatorname{Sp}(n)$ given by $\vartheta_{2}(c, f, g)=f * g$ for any fixed $c \in C_{n}(2)$. Essentially the problem arises because $\vartheta_{2}(c, f, g)$ is constant on a rather large set while for an appropriate choice of gauge any $\omega \in \mathscr{M}_{k+l}$ can be seen to be analytic. Thus we need to examine $\mathscr{M}_{k}$ more closely, which we proceed to do in the next section.

\section{The ADHM construction}

In this section we review the well-known description of $\operatorname{Sp}(n)$ instantons on $S^{4}$ in terms of linear algebra given by Atiyah, Drinfeld, Hitchin and Manin ([5] and [21], [22], [23]). Actually we summarize the more geometric version of Atiyah [2] (see also [41]), which closely parallels surface theory in Riemannian geometry, and is more visibly compatible with diagram (2.8). We then use this description in the next two sections to construct $C_{4}$ structure maps on $\mathscr{M}$. Let $E_{k}$ be a quaternionic vector bundle of (quaternionic) rank $n$ on $H P(1)=S^{4}$ associated to the principal $\operatorname{Sp}(n)$ bundle $P_{k}$. This is the quaternionic analogue of the theory of Hermitian vector bundles. Now imbedding $E_{k}$ in the trivial vector bundle $H^{k+n}$ of rank $k+n$ we obtain an exact sequence

$$
0 \rightarrow E_{k} \rightarrow H^{k+n} \rightarrow k L \rightarrow 0,
$$

where $k L$ denotes $k$ copies of the tautological (Hopf) quaternionic line bundle $L$ on $H P(1)$. Choosing the flat metric on $H^{k+n}$ with its standard flat connection $\nabla^{\prime}$, the sequence splits

$$
H^{k+n} \simeq E_{k} \oplus k L
$$

Thus restricting $\nabla^{\prime}$ to $E_{k}$ we get a map

$$
\nabla^{\prime}: \Gamma\left(E_{k}\right) \rightarrow \Gamma\left(E_{k} \otimes \bigwedge^{1} S^{4}\right) \oplus \Gamma\left(k L \otimes \bigwedge^{1} S^{4}\right)
$$

where $\Gamma\left(E_{k}\right)$ denotes the vector space of smooth sections of $E$. This map can be viewed as the analogue of Gauss' formula in surface theory. Projecting onto the first factor gives the induced connection

$$
\nabla=\pi_{1} \circ \nabla^{\prime}: \Gamma\left(E_{k}\right) \rightarrow \Gamma\left(E_{k}\right) \otimes \Gamma\left(\bigwedge^{1} S^{4}\right)
$$

on $E_{k}$, whereas projecting onto the second factor gives the analogue of the second fundamental form

$$
\Pi=\pi_{2} \circ \nabla^{\prime}: \Gamma\left(E_{k}\right) \rightarrow \Gamma(k L) \otimes \Gamma\left(\bigwedge^{1} S^{4}\right) .
$$


By choosing local bases for these vector bundles one can represent $\Pi$ by an $n \times k$ quaternionic matrix with values in the 1-forms on $S^{4}$. The analogue of Gauss' equation enables us to express the curvature $F$ of $\nabla$ in terms of the "second fundamental form" $\Pi$ (since $\nabla^{\prime}$ is flat) as follows:

$$
F=\Pi \wedge \Pi^{*} \text {. }
$$

In order to compute $F$ and its connection 1-form $A$ explicitly in terms of matrices we consider the quaternionic vector space $H^{n}$ with its standard norm

$$
\|x\|^{2}=\sum_{i=1}^{n}\left|x_{i}\right|^{2}
$$

for $x_{i} \in H$. Now the space $L\left(H^{n}, H^{m}\right)$ of quaternionic linear maps thought of as a left $H$-module is a normed linear space with the operator norm

$$
\|T\|=\sup _{\|x\| \neq 0} \frac{\|T x\|}{\|x\|}
$$

where $T \in L\left(H^{n}, H^{m}\right)$. Moreover, $L\left(H^{n}, H^{m}\right)$ is a Banach algebra in the sense that

$$
\|T S\| \leq\|T\|\|S\|
$$

Of course choosing bases in $H^{n}$ and $H^{m}$ gives an isomorphism $L\left(H^{n}, H^{m}\right) \simeq$ $M_{n, m}(H)$ where $M_{n, m}(H)$ denotes the set of quaternionic valued $n$ by $m$ matrices.

Now a choice of a smooth imbedding $u: E \rightarrow H^{k+n}$ can be thought of as a choice of a smooth classifying map $f: S^{4} \rightarrow G_{n, n+k}(H)$, where $G_{n, n+k}(H)$ is the Grassmannian of quaternionic $n$-planes in $H^{n+k}$. Locally $f$ is given by maps of the form $x \mapsto U(x)$, where $U$ denotes the affine coordinates on $G_{n, n+k}(H)$. If we choose a local symplectic basis (gauge) for $E$ and a basis for $H^{k+n}$ we can represent $u$ by an $n+k$ by $n$ quaternionic matrix which satisfies $u^{*} u=I$, where $u^{*}$ denotes the quaternionic conjugate matrix and $I$ denotes the $n$ by $n$ identity matrix. To obtain $f$ explicitly we need to give an imbedding of the normal bundle $v: k L \rightarrow H^{k+n}$ which satisfies the orthogonality condition

$$
v^{*} u=0
$$

After making appropriate choices of bases any such map can be represented in an affine chart $(H, x)$ of $H P(1)$ by

$$
v(x)=\left(\begin{array}{c}
\Lambda \\
B-x I
\end{array}\right),
$$


where $\Lambda$ is a $k$ by $n$ quaternionic matrix and $B$ is a $k$ by $k$ quaternionic matrix. Furthermore $v(x)$ satisfies the condition

$$
\operatorname{rank} v(x)=k \quad \text { for all } x \in H P(1),
$$

where rank $v(x)$ denotes the row rank of the matrix.

Viewing the components of $u$ as homogeneous coordinates on the Grassmannian we can solve $v^{*} u=0$ locally for $u$ as a function of $x$ or equivalently, in terms of affine coordinates, $U$ as a function of $x$. This gives the local expression for the classifying map

$$
f(x)=U(x)=\left(\Lambda(B-x I)^{-1}\right)^{*} .
$$

Then the connection 1-form $A$ can be obtained by pulling back the standard connection $\omega_{G}$ on $G_{n, n+k}(H)$. In local coordinates

$$
A=f^{*} \omega_{G}=\sigma U^{*} d U \sigma+\sigma^{-1} d \sigma,
$$

where $\sigma^{-2}=I+U^{*} U$. Note that the local expression for both the classifying map $f$ and the connection 1-form $A$ are not defined at the points of $H P(1)$ corresponding to the eigenvalues of $B$. These "apparent singularities" are due to a bad choice of coordinates on $G_{n, n+k}(H)$ at those points. Changing affine coordinates on the Grassmannian corresponds to mixing the $\Lambda$ and $B$ matrices so that the local expression in the new coordinates at that point is well defined.

As indicated by the previous construction we are interested in the set

$$
\mathscr{B}_{k}^{\prime}=\left\{(\Lambda, B) \in M_{n, k}(H) \times M_{k, k}(H) \text { : condition (4.12) is satisfied }\right\} .
$$

Clearly $\mathscr{B}_{k}^{\prime}$ is an open set of the normed linear space $M_{n+k, k}(H)$. The previous discussion culminating in the formula for $A$ thus describes a continuous map $\alpha_{k}^{\prime}: \mathscr{B}_{k}^{\prime} \rightarrow \mathscr{A}_{k}$. Now the real orthogonal group $\mathrm{O}(k)$ acts naturally on $M_{n+k, k}(H) \simeq M_{n, k}(H) \times M_{k, k}(H)$ by sending $(\Lambda, B) \mapsto\left(\Lambda T, T^{-1} B T\right)$ where $T \in \mathrm{O}(k)$. This clearly leaves $\mathscr{B}_{k}^{\prime}$ invariant. Thus by restriction we obtain the quotient space $\mathscr{B}_{k}=\mathscr{B}_{k}^{\prime} / \mathrm{O}(k)$. Moreover, one easily sees that changing $(\Lambda, B)$ to $\left(\Lambda T, T^{-1} B T\right)$ does not affect $A$, so we get a continuous map $\mathscr{B}_{k} \rightarrow \mathscr{A}_{k}$. Thus composing this map with the natural projection $\mathscr{A}_{k} \rightarrow \mathscr{C}_{k}$ gives a continuous map $\mathscr{B}_{k}$ to $\mathscr{C}_{k}$. Summarizing we have

Proposition 4.16. For any symplectic vector bundle $E_{k}$ over $S^{4}$ there is a continuous map $\alpha_{k}: \mathscr{B}_{k} \rightarrow \mathscr{C}_{k}$, where $\alpha_{k}$ is induced by $\alpha_{k}^{\prime}$ above.

Remarks. 1. If $\hat{\alpha}_{k}: \mathscr{B}_{k} \rightarrow \Omega^{4} B \mathrm{Sp}(n)$ is the composition of $\alpha_{k}$ and the homotopy equivalence $\mathscr{C}_{k} \simeq \Omega^{4} B \operatorname{Sp}(n)$ given in [7] then $\hat{\alpha}_{k}((\Lambda, B))=f$ where $f$ is given by (4.13).

2. The inclusion $j_{k}: C_{k}\left(R^{4}\right) \rightarrow \mathscr{M}_{k}$ in (2.8) is given by sending the $k$ distinct points in $R^{4} \cong H^{1}$ to the pair $(\Lambda, B)$, where $B$ is the diagonal matrix whose 
diagonal entries are precisely the $k$ distinct points in $H^{1}$ and $\Lambda$ is the matrix all of whose entries are 1.

We shall need an explicit formula for the curvature of the connections which are in the image of $\alpha_{k}$. This is given by Atiyah [2, II-3.11].

$$
F=N d x \rho^{-2} d \bar{x} N^{*},
$$

where $N=-u u_{2}^{*}$ in terms of the homogeneous coordinates $u^{*}=\left(u_{1}^{*}, u_{2}^{*}\right)$ on the Grassmannian. Here $N=P C$ in Atiyah's terminology [2, II-3.11], and

$$
\rho^{2}=v^{*} v=\left(B^{*}-\bar{x} I\right)(B-x I)+\Lambda^{*} \Lambda \text {. }
$$

We note that the matrix $\rho^{2}(x)$ will play an extremely important role in the analysis to follow. We have written it here explicitly (after making certain choices). Strictly speaking, $\rho^{2}$ is a section of the endomorphism bundle $\operatorname{End}(k L)$ on $S^{4} \simeq H P(1)$.

Since $d x \wedge d \bar{x}$ span the self-dual two-forms on an affine open set of $S^{4}$, the connection will be self-dual if and only if $\rho^{2}$ is real. The reality of $\rho^{2}$ is equivalent to the following two conditions [2]:

(i) $B$ is symmetric,

(ii) $B^{*} B+\Lambda^{*} \Lambda$ is real.

This leads us to define

$$
\mathscr{S}_{k}^{\prime}=\left\{(\Lambda, B) \in \mathscr{B}_{k}^{\prime}: \quad(4.19) \text { is satisfied }\right\} .
$$

Notice that conditions (4.19) are unaltered by the $\mathrm{O}(k)$-action on $\mathscr{B}_{k}^{\prime}$, giving a well-defined quotient $\mathscr{S}_{k}=\left(\mathscr{S}_{k}^{\prime}\right) / \mathrm{O}(k)$. Thus the set of $\mathrm{O}(k)$-orbits $\{\Lambda, B\}$ of matrices $(\Lambda, B)$ satisfying conditions (4.8) and (4.13) automatically give self-dual connections of instanton number $k$. What is remarkable is the theorem of Atiyah, Drinfeld, Hitchin, and Manin ([5] and [21], [22], [23]), which proves that $\mathscr{S}_{k}$ gives all instantons of charge $k$ up to gauge equivalence under the based gauge group. The proof requires the full algebro-geometric machinery of stable algebraic vector bundles on $C P(3)$ and the monad construction of Horrocks and Barth ([29], [9], [10]). We rephrase the ADHM theorem for $G=\operatorname{Sp}(n)$ as

Theorem 4.20. For a principal $\mathrm{Sp}(n)$-bundle, $\mathscr{S}_{k}$ and $\mathscr{M}_{k}$ are homeomorphic.

Proof. Define the map $\delta_{k}: \mathscr{S}_{k} \rightarrow \mathscr{M}_{k}$ by the previous construction which assigns to each equivalence class $\{\Lambda, B\}$ a self-dual connection. That $\delta_{k}$ is a bijection is the ADHM theorem in the form described by Atiyah [2, p. 26] for $\operatorname{Sp}(1)$ and generalized to $\operatorname{Sp}(n)[2, \mathrm{p} .27]$. The continuity of $\delta_{k}$ follows easily 
from the continuity of the map $\alpha_{k}$ of Proposition 4.16. To see the continuity of the inverse map, recall that [7],

$$
C_{k} \simeq \Omega^{3} G \simeq \Omega^{4} B G \subset \operatorname{Map}\left(S^{4}, B G\right)
$$

with the compact-open topology. From the ADHM construction the maps $S^{4} \rightarrow B G$ given by $x \mapsto U(x)$ in (4.13) factor through the Grassmannian $G_{n, n+k}(H)$ and thus are given locally by rational maps. Moreover, on the mapping space $\operatorname{Map}\left(S^{4}, G_{n, n+k}(H)\right)$ the compact-open topology coincides with the uniform metric topology and one easily sees that $\delta_{k}^{-1}$ is continuous. This completes the proof.

In the succeeding sections we shall identify $\mathscr{S}_{k}$ with $\mathscr{M}_{k}$ without explicit reference to Theorem 4.20 and no longer write $\mathscr{S}_{k}$.

Finally we close this section by mentioning briefly the full (unbased) moduli space $\mathscr{M}_{k}^{\prime} \simeq \mathscr{M}_{k} / G$ where $G=\operatorname{Sp}(n) / Z_{2}$. Actually it is the quotient space $\mathscr{M}_{k}^{\prime}$ which appears in Atiyah's theorem [2, p. 26]. It is shown there, for all $\operatorname{Sp}(n)$, that $\mathscr{M}_{k}^{\prime}$ can be identified with the set of equivalence classes of pairs $(\Lambda, B)$ under the action of $\operatorname{Sp}(n) \times \mathrm{O}(k)$ given by sending $(\Lambda, B)$ to $\left(q \Lambda T, T^{-1} B T\right)$ where $q \in \operatorname{Sp}(n)$ and $T \in \mathrm{O}(k)$. As mentioned previously in $\S 1$, for $n=1$, $\mathscr{M}_{k} \rightarrow \mathscr{M}_{k}^{\prime}$ is a principal $\mathrm{Sp}(1) / Z_{2} \simeq \mathrm{SO}(3)$ bundle, but for $n \neq 1$ it is not a principal fibration owing to the presence of reducible connections.

\section{The loop sum map on instantons}

In this section we give one of the key technical results of this paper, namely the construction of a "loop sum" map

$$
\text { *: } \mathscr{M}_{k} \times \mathscr{M}_{l} \rightarrow \mathscr{M}_{k+l},
$$

which is homotopy compatible with the loop sum map on $\Omega^{4} B \operatorname{Sp}(n)$. Actually we obtain a 1-parameter family of such homotopy loop sum maps as stated in the following theorem.

Theorem 5.2. There is a continuous one-parameter family of continuous maps

$$
\vartheta_{t}: \mathscr{M}_{k} \times \mathscr{M}_{l} \rightarrow \mathscr{M}_{k+l}
$$

such that for each $0<t \leq 1$ the following diagram homotopy commutes:

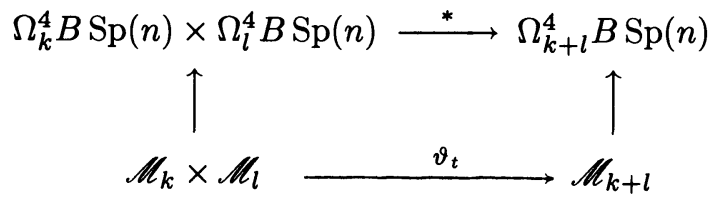


where the vertical arrows are given by the compositions of the natural inclusions $i_{j}$ with any homotopy equivalence of $\mathscr{C}_{j} \rightarrow \Omega_{j}^{4} B \mathrm{Sp}(n)$, and * is the standard loop sum map on $\Omega^{4} B \operatorname{Sp}(n)$.

The remainder of this section is devoted to the construction of the maps $\vartheta_{t}$, while the proof that diagram (5.4) homotopy commutes is given in $\S 6$. In order to construct the maps $\vartheta_{t}$ we construct maps from $\mathscr{M}_{k} \times \mathscr{M}_{l}$ to the Taubes tubular neighborhood $\mathscr{C}_{k+l}^{\varepsilon}$ of $\mathscr{M}_{k+l}$ (recall Theorem 2.9). More precisely, we define $\mathscr{B}_{k}^{\varepsilon}=\alpha_{k}^{-1}\left(\mathscr{C}_{k}^{\varepsilon}\right)$, and construct a 1-parameter family of maps $\phi_{t}: \mathscr{M}_{k} \times$ $\mathscr{M}_{l} \rightarrow \mathscr{B}_{k+l}^{\varepsilon}$. Then using the strong deformation retraction $\mathscr{T}_{k+l}: \mathscr{C}_{k+l}^{\varepsilon} \rightarrow$ $\mathscr{M}_{k+l}$ given in 2.9 and Proposition 4.16 we obtain the following commutative diagram:

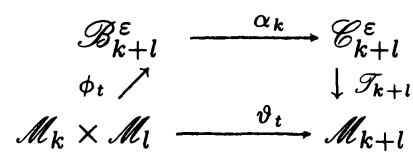

Hence the construction of the maps $\vartheta_{t}$ is equivalent to the construction of the $\operatorname{maps} \phi_{t}$.

We begin by defining a map from $\mathscr{M}_{k}$ to itself. To set our notation we let $\hat{b}_{k}$ denote the pair $(\Lambda, B) \in \hat{\mathscr{B}}_{k}$ and $b_{k}$ its equivalence class in $\mathscr{B}_{k}$. Notice that the norm

$$
\left\|b_{k}\right\|=\sup _{\|u\|=1}\|(\Lambda, B) u\|
$$

is independent of its representative $\left(\Lambda T, T^{-1} B T\right)$ for $T \in \mathrm{O}(k)$. Now for each $\delta \in(0,1]$ we define maps $\hat{\psi}_{\delta}^{ \pm}: \hat{\mathscr{B}}_{k} \rightarrow M_{k, n}(H) \times M_{k, k}(H)$ by

$$
\hat{\psi}_{\delta}^{ \pm}(\Lambda, B)=\left(\frac{\delta}{\left\|b_{k}\right\|} \Lambda, \pm I+\frac{\delta}{\left\|b_{k}\right\|} B\right) .
$$

Clearly the $\hat{\psi}_{\delta}^{ \pm}$are continuous.

Lemma 5.7. For each $\delta \in(0,1]$ the maps $\hat{\psi}_{\delta}^{ \pm}$are contractions from $\hat{\mathscr{B}}_{k}$ to itself. Furthermore, formula (5.6) gives continuous maps $\hat{\psi}_{\delta}^{ \pm}: \mathscr{B}_{k} \rightarrow \mathscr{B}_{k}$ which leave the subspace $\mathscr{M}_{k}$ invariant and depend continuously on $\delta$. Thus for any $\delta, \delta^{\prime} \in(0,1], \hat{\psi}_{\delta}^{ \pm}$and $\hat{\psi}_{\delta^{\prime}}^{ \pm}$are homotopic.

Proof. One easily checks that the $\hat{\psi}_{\delta}^{ \pm}$are $\mathrm{O}(k)$-equivariant and thus define maps $\psi_{\delta}^{ \pm}$on the quotient $\mathscr{B}_{k}$ which are clearly continuous. To see that $\mathscr{M}_{k}$ is invariant we notice that conditions (4.19) are preserved by $\hat{\psi}_{\delta}^{ \pm}$. Thus $\hat{\psi}_{\delta}^{ \pm}$ restrict to self-maps on $\mathscr{M}_{k}$ which are continuous functions of $\delta$. For $0<\delta<$ $\delta^{\prime} \leq 1$ the homotopy on $\hat{\mathscr{B}}_{k}$ is given by

$$
\hat{\psi}_{\delta, \delta^{\prime}}^{ \pm}(t)=\left(\frac{t \delta^{\prime}+(1-t) \delta}{\left\|b_{k}\right\|} \Lambda, \pm I+\frac{t \delta^{\prime}+(1-t) \delta}{\left\|b_{k}\right\|} B\right),
$$

which gives a homotopy on the quotient $\mathscr{B}_{k}$. q.e.d. 
We next define a "diagonal imbedding"

$$
\begin{gathered}
\left(M_{k, n}(H) \times M_{k, k}(H)\right) \times\left(M_{l, n}(H) \times M_{l, l}(H)\right) \\
\stackrel{\Delta}{\longrightarrow} M_{k+l, n}(H) \times M_{k+l, k+l}(H),
\end{gathered}
$$

which is the natural identification of $M_{k, n}(H) \times M_{l, n}(H)$ with $M_{k+l, n}(H)$ on the first factors and the diagonal imbedding

$$
M_{k, k}(H) \times M_{l, l}(H) \rightarrow M_{k+l, k+l}(H)
$$

on the second factors. We compose $\Delta$ with the maps (5.5) giving maps

$$
\hat{\phi}_{\delta}=\Delta \circ\left(\hat{\psi}_{\delta}^{+} \times \hat{\psi}_{\delta}^{-}\right): \hat{\mathscr{B}}_{k} \times \hat{\mathscr{B}}_{l} \rightarrow M_{k+l, n}(H) \times M_{k+l, k+l}(H) .
$$

Lemma 5.11. For $0<\delta<1$ the image $\hat{\phi}_{\delta}\left(\hat{\mathscr{B}}_{\delta} \times \hat{\mathscr{B}}_{l}\right)$ lies in $\hat{\mathscr{B}}_{k+l}$ and the eigenvalues of $\hat{\phi}_{\delta}\left(b_{k}, b_{l}\right)$ for any $\left(b_{k}, b_{l}\right) \in \hat{\mathscr{B}}_{k} \times \hat{\mathscr{B}}_{l}$ lie in open discs of radius $\delta$ centered about $x= \pm 1$. Furthermore, $\hat{\phi}_{\delta}$ are contractions and define continuous maps $\phi_{\delta}: \mathscr{B}_{k} \times \mathscr{B}_{l} \rightarrow \mathscr{B}_{k+l}$ on the quotient space. For any $\delta, \delta^{\prime} \in(0,1], \phi_{\delta}$ and $\phi_{\delta^{\prime}}$ are homotopic.

Proof. To see that $\hat{\phi}_{\delta}\left(\hat{\mathscr{B}}_{k} \times \hat{\mathscr{B}}_{l}\right) \subset \hat{\mathscr{B}}_{k+l}$ we need only to check the rank condition (4.12). It suffices to show that no $x \in H P(1)$ can be a simultaneous eigenvalue of the $B$ matrix in $\psi_{\delta}^{+}\left(\Lambda_{k}, B_{l}\right)$ and $\psi_{\delta}^{-}\left(\Lambda_{l}, B_{l}\right)$. Let $x_{k}$ be an eigenvalue of $I+\left(\delta /\left\|b_{k}\right\|\right) B_{k}$ and $x_{l}$ an eigenvalue of $-I+\left(\delta /\left\|b_{l}\right\|\right) B_{l}$. Then there are eigenvectors $\xi_{k} \in H^{k}$ and $\xi_{l} \in H^{l}$ which we can assume are of unit norm, such that

$$
\left(x_{k}-1\right) \xi_{k}=\frac{\delta}{\left\|b_{k}\right\|} B_{k} \xi_{k}, \quad\left(x_{l}+1\right) \xi_{l}=\frac{\delta}{\left\|b_{l}\right\|} B_{l} \xi_{l} .
$$

Thus taking norms and using the facts that the rank condition (4.12) guarantees $\left\|B_{k}\right\|<\left\|b_{k}\right\|$ and that $\hat{\psi}_{\delta}^{ \pm}$is a contraction we get $\left|x_{k}-1\right|<\delta \leq 1$ and $\left|x_{l}+1\right|<\delta \leq 1$ so $x_{k} \neq x_{l}$. This also shows that all the eigenvalues lie within open discs of radius $\delta$ about \pm 1 . The fact that $\hat{\phi}_{\delta}$ are contractions follows immediately from the fact that $\hat{\psi}_{\delta}^{ \pm}$are contractions and that $\Delta$ is a natural inclusion of linear spaces.

That the $\hat{\phi}_{\delta}$ pass to continuous maps on the quotient spaces follow from the fact that $\Delta$ intertwines the $\mathrm{O}(k) \times \mathrm{O}(l)$ action on $\hat{\mathscr{B}}_{k} \times \hat{\mathscr{B}}_{l}$ with its action under the diagonal imbedding $\mathrm{O}(k) \times \mathrm{O}(l) \rightarrow \mathrm{O}(k+l)$ on $\hat{B}_{k+1}$. Finally that $\phi_{\delta}$ and $\phi_{\delta^{\prime}}$ are homotopic follows from Lemma 5.7 and the continuity of $\Delta$. q.e.d.

We note in passing the fact that $\hat{\phi}_{\delta}$ passing to continuous maps on the quotient spaces depends on the fact that we are quotienting out only by the based gauge group. If one quotients out by the full gauge group (as described in the last paragraph of $\S 4$ ) then the $\hat{\phi}_{\delta}$ do not pass to well-defined maps on the $\mathscr{M}^{\prime}$ level. 
We are interested in the maps $\phi_{\delta}$ restricted to $\mathscr{M}_{k} \times \mathscr{M}_{l} \subset \mathscr{B}_{k} \times \mathscr{B}_{l}$. Let $b_{k} \in \mathscr{M}_{k}$ and $b_{l} \in \mathscr{M}_{l}$. Although $b_{k}$ and $b_{l}$ are instantons the image $\phi_{\delta}\left(b_{k}, b_{l}\right)$ need not be an instanton for any $\delta$, but for $\delta$ small enough $\phi_{\delta}\left(b_{k}, b_{l}\right)$ will always be an "almost" instanton. We now make this precise.

Now consider the section $\rho^{2}$ of $\operatorname{End}_{H} k L$ defined in (4.18). For $\hat{b}_{k}=$ $\left(\Lambda_{k}, B_{k}\right) \in \hat{S}_{k}$ and $\hat{b}_{l}=\left(\Lambda_{l}, B_{l}\right) \in \hat{S}_{l}, \rho^{2} \circ \hat{\phi}_{\delta}$ represents a smooth section of the bundle $\operatorname{End}_{H}(k+l) L$, which is isomorphic to

$$
\operatorname{End}_{H} k L \oplus \operatorname{End}_{H} l L \oplus \operatorname{Hom}_{H}(k L, l L) \oplus \operatorname{Hom}_{H}(l L, k L) .
$$

Choosing affine coordinates $x$ on $H P(1)$ and local frames for $k L$ and $l L$, we can represent $\rho^{2} \circ \hat{\phi}_{\delta}$ as a matrix

$$
\left(\begin{array}{cc}
S_{k}^{+}(\delta, x) & \delta^{2} E_{k l} \\
\delta^{2} E_{k l}^{*} & S_{l}^{-}(\delta, x)
\end{array}\right)
$$

where

$$
\begin{gathered}
S_{k}^{ \pm}(\delta, x)=\left(\frac{\delta}{\left\|b_{k}\right\|} B_{k}^{*}-(\bar{x} \mp 1) I\right)\left(\frac{\delta}{\left\|b_{k}\right\|} B_{k}-(x \mp 1) I\right) \\
+\frac{\delta^{2}}{\left\|b_{k}\right\|^{2}} \Lambda_{k}^{*} \Lambda_{k}, \\
E_{k l}=\frac{1}{\left\|b_{k}\right\|\left\|b_{l}\right\|} \Lambda_{k}^{*} \Lambda_{l} .
\end{gathered}
$$

If we choose a different representative $\left(\Lambda_{k} T_{k}, T_{k}^{-1} B_{k} T_{k}\right)$ for $b_{k} \in M_{k}$ where $T_{k} \in \mathrm{O}(k)$, then

$$
\begin{aligned}
S_{k}^{ \pm}(\delta, x) & \mapsto T_{k}^{-1} S_{k}^{ \pm}(\delta, x) T_{k}, \\
E_{k l} & \mapsto T_{k}^{-1} E_{k l} T_{l}, \\
\rho^{2} \circ \hat{\phi}_{\delta} & \mapsto T_{k+l}^{-1} \rho^{2} \circ \hat{\phi}_{\delta} T_{k+l},
\end{aligned}
$$

where $T_{k+l}$ is the image of $\left(T_{k}, T_{l}\right)$ under the diagonal imbedding $\mathrm{O}(k) \times$ $\mathrm{O}(l) \rightarrow \mathrm{O}(k+l)$.

Lemma 5.16. The matrices $S_{k}^{ \pm}(\delta, x)$ and $E$ satisfy

(i) $S_{k}^{ \pm}(\delta, x)$ is a real invertible self-adjoint matrix for all $\delta \in(0,1]$ and all $x \in H$.

(ii) $\left\|S_{k}^{ \pm}(\delta, x)\right\|<\left(|x \mp 1|^{2}+2 \delta^{2}\right)$.

(iii) $\|E\|<1$.

Furthermore, these conditions are independent of the choice of representative $\left(\hat{b}_{k}, \hat{b}_{l}\right)$ of $\left(b_{k}, b_{l}\right) \in \mathscr{M}_{k} \times \mathscr{M}_{l}$.

Proof. (i) follows from the fact that $\hat{b}_{k}$ represents an instanton and thus satisfies conditions (4.12) and (4.19). The remainder of lemma is easily verified and left to the reader. 
Lemma 5.17. For each representative $\left(\hat{b}_{k}, \hat{b}_{l}\right) \in \hat{\mathscr{S}}_{k} \times \hat{\mathscr{S}}_{l}$ the matrix $\rho^{2} \circ \hat{\phi}_{\delta}\left(\hat{b}_{k}, \hat{b}_{l}\right)$ is invertible for all $0<\delta \leq 1$ and for all $x \in H$.

Proof. This follows immediately from Lemma 5.11 and the explicit form of $\rho^{2} \circ \hat{\phi}_{\delta}\left(\hat{b}_{k}, \hat{b}_{l}\right)$.

We need estimates on $\left\|\operatorname{Im} \rho^{-2} \circ \phi_{\delta}\right\|$. The following lemma shows that although $\rho^{2} \circ \phi_{\delta}$ is singular as $\delta \rightarrow 0$ the limit of $\operatorname{Im} \rho^{-2} \circ \phi_{\delta}$ is well defined at $\delta=0$. We may write $\rho^{2} \circ \phi_{\delta}=R(\delta, x)+\delta^{2} F$, where $R(\delta, x)$ is real and denotes the block diagonal entries in (5.13) and $\delta^{2} F$ denotes the off diagonal blocks, where we note that $F$ is independent of both $\delta$ and $x$. However $F$ may contain both real and imaginary entries and we now rewrite

$$
\rho^{-2} \circ \phi_{\delta}=\mathfrak{R}(\delta, x)+\mathfrak{I}(\delta, x),
$$

where $\mathfrak{R}$ and $\mathfrak{I}$ denote the real and imaginary parts of $\rho^{-2} \circ \phi_{\delta}$ respectively.

Lemma 5.19. There are constant matrices $\mathfrak{I}(0, \pm I)$ such that

$$
\lim _{\delta \rightarrow 0} \mathfrak{I}(\delta, x)= \begin{cases}\mathfrak{I}(0,1) & \text { if } x=1 \\ 0 & \text { if } x \neq \pm 1 \\ \mathfrak{I}(0,-1) & \text { if } x=-1\end{cases}
$$

for all $x \in H$.

Proof. For $\delta \in(0,1], \rho^{-2} \circ \phi_{\delta}$ is invertible so

$$
\begin{gathered}
I=\left(R+\delta^{2} F_{0}\right) \mathfrak{R}+\delta^{2}\left(F_{1} \mathfrak{I}\right)_{0}, \\
0=\delta^{2} F_{1} \Re+\left(R+\delta^{2} F\right) \mathfrak{I},
\end{gathered}
$$

where $A=A_{0}+A_{1}$ denotes the decomposition of a quaternionic matrix into its real and imaginary parts. From these equations we have

$$
\begin{aligned}
\mathfrak{I}= & -\delta^{2}\left(I+\delta^{2} R^{-1} F\right)^{-1} R^{-1} F_{1} R^{-1} R \\
& \times\left(I+\delta^{2} R^{-1} F_{0}\right)^{-1} R^{-1}\left(I-\delta^{2}\left(F_{1} \mathfrak{I}\right)_{0}\right) .
\end{aligned}
$$

Now each of the following terms has a well-defined limit as $\delta \rightarrow 0$ :

$$
\begin{aligned}
& \lim _{\delta \rightarrow 0}\left(I+\delta^{2} R^{-1} F\right)^{-1}= \begin{cases}I-A_{k} & \text { if } x=1, \\
I & \text { if } x \neq \pm 1, \\
I-A_{l} & \text { if } x=-1,\end{cases} \\
& \lim _{\delta \rightarrow 0} \delta^{2} R^{-1} F R^{-1}= \begin{cases}\frac{1}{4}\left(A_{k}+A_{k}^{*}\right) & \text { if } x=1, \\
0 & \text { if } x \neq \pm 1, \\
\frac{1}{4}\left(A_{l}+A_{l}^{*}\right) & \text { if } x=-1,\end{cases} \\
& \lim _{\delta \rightarrow 0} R\left(I+\delta^{2} R^{-1} F\right)^{-1} R^{-1}= \begin{cases}I-A_{k}^{*} & \text { if } x=1, \\
I & \text { if } x \neq \pm 1, \\
I-A_{l}^{*} & \text { if } x=-1,\end{cases}
\end{aligned}
$$


where

$$
\begin{aligned}
A_{k} & =\left(\begin{array}{cc}
0 & \left(B_{k}^{*} B_{k}+\Lambda_{k}^{*} \Lambda_{k}\right)^{-1} E \\
0 & 0
\end{array}\right)\left\|b_{k}\right\|^{2}, \\
A_{l} & =\left(\begin{array}{cc}
0 & 0 \\
\left(B_{l}^{*} B_{l}+\Lambda_{l}^{*} \Lambda_{l}\right)^{-1} E^{*} & 0
\end{array}\right)\left\|b_{l}\right\|^{2} .
\end{aligned}
$$

The result now follows by taking the limit of (5.22). q.e.d.

In order to get explicit bounds on $\mathscr{Y} \mathscr{M}\left(\phi_{\delta}\left(b_{k}, b_{l}\right)\right)$ we define $D^{ \pm}(r)=\{x \in$ $H:|x \mp 1| \leq r\}$, and let $H^{\prime}$ be the complement of $D^{+}(r) \cup D^{-}(r)$ in $H$.

Lemma 5.23. For all $x \in H^{\prime}$ there is a $\delta_{1}>0$, independent of $\left(b_{k}, b_{l}\right)$, such that

$$
\|\mathfrak{I}(\delta, x)\|<2 \delta^{2} / r^{4}
$$

for all $\delta \in\left(0, \delta_{1}\right]$.

Proof. First we rewrite equation (5.22) as

$$
\mathfrak{I}=-\delta^{2}\left(R+\delta^{2} F\right)^{-1} F_{1}\left(R+\delta^{2} F_{0}\right)^{-1}\left(I-\delta^{2}\left(F_{1} \mathfrak{I}\right)_{0}\right) .
$$

Since $F$ is independent of $x, \lim _{\delta \rightarrow 0}\left(R+\delta^{2} F\right)^{-1}$ exists and

$$
\lim _{|x| \rightarrow \infty} R^{-1}(\delta, x)=0
$$

hence $\left(R(\delta, x)+\delta^{2} F\right)^{-1}$ is uniformly continuous on $[0,1] \times H^{\prime}$. Thus

$$
s(\delta)=\sup _{x \in H^{\prime}}\left\|\left(R+\delta^{2} F\right)^{-1}\right\|<\infty
$$

is continuous and it follows, using Lemma 5.16, that

$$
\|\mathfrak{I}(\delta, x)\|<\delta^{2} s^{2}\left(1+\delta^{2}\|\mathfrak{I}\|\right) .
$$

But now an explicit computation gives

$$
\begin{aligned}
\lim _{\delta \rightarrow 0} s(\delta) & \left.\left.=\lim _{\delta \rightarrow 0} \sup _{x \in H^{\prime}} \|\left(R+\delta^{2} F\right)^{-1}\right)\left\|=\sup _{x \in H^{\prime}} \lim _{\delta \rightarrow 0}\right\|\left(R+\delta^{2} F\right)^{-1}\right) \| \\
& =\sup _{x \in H^{\prime}}\left\|\left(\begin{array}{cc}
|x-1|^{-2} I & 0 \\
0 & |x+1|^{-2} I
\end{array}\right)\right\| \\
& =r^{-2} .
\end{aligned}
$$

Thus after multiplying (5.25) by $r^{4}$ there is, by the continuity of $s(\delta)$, a $\delta_{1} \in(0,1]$ such that

$$
\frac{r^{4}}{\sqrt{2}}\|\mathfrak{I}\|<\left(r^{4}-\delta^{4} s^{2}(\delta) r^{4}\right)\|\mathfrak{I}\|<\delta^{2} r^{4} s^{2}(\delta)<\sqrt{2} \delta^{2},
$$

whenever $\delta \in\left(0, \delta_{1}\right]$. Furthermore, since $\left(R+\delta^{2} F\right)^{-1}$ is well defined as $\left\|b_{k}\right\|\left\|b_{l}\right\| \rightarrow 0, \delta_{1}$ can be taken to be independent of $\left(b_{k}, b_{l}\right)$. This completes the proof of the lemma. 
Lemma 5.27. For all $(\delta, x) \in\left[0, \delta_{1}\right] \times\left(D^{+}(r) \cup D^{-}(r)\right)$ there is a constant $c_{0}$ such that

$$
\|\mathfrak{I}(\delta, x)\| \leq c_{0} .
$$

Proof. $\mathfrak{I}(\delta, x)$ is continuous on $\left(0, \delta_{1}\right] \times\left(D^{+}(r) \cup D^{-}(r)\right)$. Moreover, by Lemma 5.19 , the limit exists as $\delta \rightarrow 0$. Thus

$$
c_{0}=\sup \left\{\|\mathfrak{I}(\delta, x)\|:(\delta, x) \in\left[0, \delta_{1}\right] \times\left(D^{+}(r) \cup D^{-}(r)\right)\right\}
$$

is finite.

Remark. The constant $c_{0}$ depends on the pair $\left(b_{k}, b_{l}\right)$.

Lemma 5.28. Given $\varepsilon>0$ there is a $\delta_{0}\left(b_{k}, b_{l}\right)>0$ such that for all $0<\delta<\delta_{0}\left(b_{k}, b_{l}\right), \phi_{\delta}\left(b_{k}, b_{l}\right) \in \mathscr{B}_{k+l}^{\varepsilon}$.

Proof. Given $\left(b_{k}, b_{l}\right) \in \mathscr{M}_{k} \times \mathscr{M}_{l}, \phi_{\delta}\left(b_{k}, b_{l}\right)$ lies in $\mathscr{B}_{k+l}$ for each $\delta \in(0,1]$ and thus composing with $\alpha_{k+l}$ defines a connection in $\mathscr{C}_{k+l}$. Let $F^{\phi_{\delta}}$ denote the curvature of this connection. Decomposing $F^{\phi_{\delta}}$ into its self-dual, $F_{+}^{\phi_{\delta}}$, and anti-self-dual, $F_{-}^{\phi_{\delta}}$, parts we show that given $\varepsilon>0$ there is a $\delta_{0}>0$ such that

$$
\left\|F_{-}^{\phi_{\delta}}\right\|_{2}^{2}<\varepsilon
$$

for all $0<\delta \leq \delta_{0}$, where

$$
\left\|F_{-}^{\phi_{6}}\right\|_{2}^{2}=\int_{S^{4}}\left\|F_{-}^{\phi_{\delta}}\right\|^{2}
$$

is the Hodge norm. Let us write $\left\|F_{-}^{\phi_{\delta}}\right\|_{2}^{2}$ as

$$
\int_{H^{\prime}}\left\|F_{-}^{\phi_{\delta}}\right\|^{2}+\int_{D^{+}(r) \cup D^{-}(r)}\left\|F_{-}^{\phi_{\delta}}\right\|^{2} .
$$

To estimate $\left\|F_{-}^{\phi_{\delta}}\right\|$ recall that this norm is the Hodge norm on 2 -forms and the Killing norm $\kappa(A, A)=-\operatorname{tr}\left(\operatorname{ad}(A)^{2}\right)$ on $\operatorname{sp}(n)$. Thus viewing $A \in \operatorname{sp}(n)$ as a quaternionic endomorphism, there is a constant $c_{1}$ such that

$$
\left\|F_{-}^{\phi_{\delta}}\right\|^{2} \leq c_{1}\|\Im(\delta, x)\|^{2} \operatorname{vol},
$$

where vol denotes the volume form on $S^{4}$. Thus by Lemmas 5.23 and 5.27 given $\varepsilon>0$ there are positive constants $c_{2}$ and $c_{3}$ so that

$$
\left\|F_{-}^{\phi_{\delta}}\right\|_{2}^{2}=\frac{c_{2} \delta^{4}}{r^{8}}+c_{3} r^{4}<\varepsilon
$$

by choosing $r=\left(\varepsilon / 2 c_{3}\right)^{1 / 4}$ and $\delta_{0}=\min \left\{\delta_{1},\left(\varepsilon^{3} / 8 c_{2} c_{3}^{2}\right)^{1 / 4}\right\}$. q.e.d.

To complete the existence part of the proof of Theorem 5.2, we now construct the maps $\phi_{t}: \mathscr{M}_{k} \times \mathscr{M}_{l} \mapsto \mathscr{M}_{k+l}$ of diagram (5.5). Notice that $\delta_{0}\left(b_{k}, b_{l}\right)$ is a positive valued function which is continuous in the matrix entries of $b_{k}$ 
and $b_{l}$ and $\left\|F_{-}^{\phi_{\delta}}\right\|_{2}$ is monotonically decreasing as $\delta<\delta_{0}\left(b_{k}, b_{l}\right)$ goes to zero. Thus if we define

$$
\phi_{t}\left(b_{k}, b_{l}\right)=\phi_{t \delta_{0}\left(b_{k}, b_{l}\right)}\left(b_{k}, b_{l}\right)
$$

for $0<t \leq 1$, then from (5.29) we have $\left\|F_{-}^{\phi_{t}}\right\|_{2}^{2}<\varepsilon$ for all $t \in(0,1]$.

\section{The little cubes action on instantons}

We now extend the loop sum maps $*: \mathscr{M}_{k} \times \mathscr{M}_{l} \rightarrow \mathscr{M}_{k+l}$ defined in $\S 5$ to maps

$$
\vartheta: C_{4}(p) \times \Sigma_{p}\left(\mathscr{M}_{k}\right)^{p} \rightarrow \mathscr{M}_{p k},
$$

which will permit us to define homology operations in $\mathscr{M}$.

We think of little cubes in $I^{4}$ as big cubes in $R^{4}$ in the obvious way. Fix a homeomorphism of $I^{4}$ with $H^{1}=R^{4}$. Then a point in $C_{4}(p)$ is equivalent under this fixed homeomorphism with $p$ disjoint open cubes in $H^{1}$ (with sides parallel to the axes). Let $q_{1}, \cdots, q_{p}$ denote the centers (the points whose coordinates are given by the midpoints of each side) of the $p$ disjoint cubes and let $e_{i, j}=d_{H}\left(q_{i}, q_{j}\right)$ for $i \neq j$ be the distance between the distinct centers. Thus $\hat{e}=\min _{i \neq j}\left(e_{i, j}\right)>0$ and we let $e=\max (1,1 / \hat{e})$.

Definition 6.2. Let $b_{i}=\left(\Lambda_{i}, B_{i}\right) \in \mathscr{M}_{k}$ for $1 \leq i \leq p$. Then

$$
\phi_{\delta}\left(c_{12 \cdots p}, b_{1}, \cdots, b_{p}\right)=b_{\delta}=\left(\Lambda_{\delta}, B_{\delta}\right) \in \mathscr{B}_{p k},
$$

where

(a) $\Lambda_{\delta}=(\delta / e)\left(\Lambda_{1} /\left\|b_{1}\right\|, \cdots,\left(\Lambda_{p}\right) /\left\|b_{p}\right\|\right)$,

(b) $B_{\delta}=\operatorname{diag}\left(q_{1} I+(\delta / e) B_{1}\left\|b_{1}\right\|, \cdots, q_{p} I+(\delta / e) B_{p}\left\|b_{p}\right\|\right)$, the $p k$ by $p k$ block diagonal matrix with the $k$ by $k$ matrix $q_{i} I+(\delta / e) B_{i}\left\|b_{i}\right\|$ in the $i$ th diagonal block,

(c) the $q_{i}$ 's and $e$ are uniquely determined from $c_{12 \ldots p} \in C_{4}(p)$ in the manner described in the paragraph above.

Remarks. 1. When $p=2$ and $c_{1,2}$ is fixed with $q_{1}=1$ and $q_{2}=-1$ we recover the loop sum map $\phi_{\delta}$ of $\S 5$ (with $k=l$ ).

2. We must check that $\phi_{\delta}$ is well defined. First if we consider transformations represented by $T_{1}, \cdots, T_{p}$ in $\mathrm{O}(k)$ then we need to check that

$$
\phi_{\delta}\left(c,\left(\Lambda_{1}, B_{1}\right), \cdots,\left(\Lambda_{p}, B_{p}\right)\right)
$$

is equivalent to

$$
\phi_{\delta}\left(c,\left(\Lambda_{1} T_{1}, T_{1}^{-1} B_{1} T_{1}\right), \cdots,\left(\Lambda_{p} T_{p}, T_{p}^{-1} B_{p} T_{p}\right)\right)
$$


in $\mathscr{B}_{p k}$. But this follows immediately from the fact that the map $\phi_{\delta}$ intertwines the $\mathrm{O}(k)^{p}$ action on $\left(\mathscr{M}_{k}\right)^{p}$ with its action under the diagonal imbedding $\mathrm{O}(k)^{p} \mapsto \mathrm{O}(p k)$ on $\mathscr{B}_{p k}$. Secondly we must show Definition 6.2 is $\Sigma_{p}$ equivariant; that is, that

$$
\phi_{\delta}\left(c \sigma,\left(\Lambda_{1}, B_{1}\right), \cdots,\left(\Lambda_{p}, B_{p}\right)\right)
$$

is equivalent to

$$
\phi_{\delta}\left(c,\left(\Lambda_{\sigma(1)}, B_{\sigma(1)}\right), \cdots,\left(\Lambda_{\sigma(p)}, B_{\sigma(p)}\right)\right)
$$

for all $\sigma \in \Sigma_{p}$. This follows immediately from the fact that there are elements of $\mathrm{O}(p k)$ which simultaneously permute the $p(k$ by $k$ ) block diagonal matrices in $B_{\delta}$ and $p\left(k\right.$ by $n$ ) block matrices in $\Lambda_{\delta}$.

Again we point out that this construction is not well defined if we mod out by the full gauge group (see the last paragraph of $\$ 4$ ) and thus our construction of the $\phi_{\delta}$ maps does not descend to the $\mathscr{M}^{\prime}$ level.

It follows by exactly the same arguments as in $\S 5$ that there exists $\delta_{0}>0$ (depending continuously on $\varepsilon$ and on the entries in the $b_{i}$ ) such that for all $0<t<1, \phi_{t \delta_{0}}: C_{4}(p) \times_{\Sigma_{p}}\left(\mathscr{M}_{k}\right)^{p} \rightarrow \mathscr{B}_{p k}$ maps into $\mathscr{B}_{p k}^{\varepsilon}$ and for appropriate choice of $\varepsilon$ we may compose with the Taubes strong deformation retraction to obtain our desired maps $\vartheta_{t}: C_{4}(p) \times_{\Sigma_{p}}\left(\mathscr{M}_{k}\right)^{p} \rightarrow M_{p k}$. Again as in $\S 5$ it is routine to verify that for any $t_{1}, t_{2}$ such that $0<t_{1} \leq t_{2} \leq 1$ we have that $\vartheta_{t_{1}}$ is homotopic to $\vartheta_{t_{2}}$. We are interested here in the maps $\vartheta_{t}$ only up to homotopy and thus will write $\vartheta$ to denote the generic $\vartheta_{t}$ for $0<t \leq 1$.

Now $(\mathscr{M}, \vartheta)$ is not a $C_{4}$ operad space in that the analog of diagram (3.5) commutes only up to homotopy but does not strictly commute. However our structure maps $\vartheta: C_{4}(p) \times \Sigma_{p}\left(\mathscr{M}_{k}\right)^{p} \rightarrow \mathscr{M}_{p k}$ are sufficiently well behaved, up to homotopy, as demonstrated in the following two theorems, the first of which completes the proof of Theorem 5.2.

Theorem 6.3. The following diagram commutes up to homotopy:

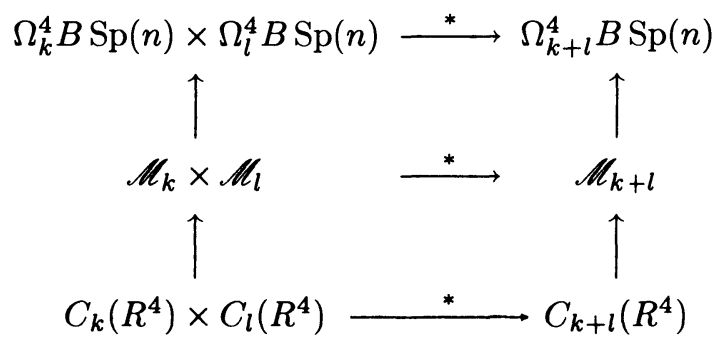

Proof. We show both the squares in (6.4) commutes up to homotopy.

The upper square: Our model for $S^{4}$ is $H \cup \infty$ and hence our model for the loop sum can be to write $x=x_{0}+x_{1} i+x_{2} j+x_{3} k$ in $H$ and use the 
real coordinate $x_{0}$ as the suspension coordinate. More precisely for all $f, g \in$ $\Omega^{4} B \operatorname{Sp}(n)$ we have

$$
f * g(x)= \begin{cases}f\left(\ln x_{0}+x_{1} i+x_{2} j+x_{3} k\right) & \text { if } x_{0} \geq 0 \\ g\left(\ln \left(-1 / x_{0}\right)+x_{1} i+x_{2} j+x_{3} k\right) & \text { if } x_{0} \leq 0 .\end{cases}
$$

Let $b_{1}=\left(\Lambda_{1}, B_{1}\right) \in \mathscr{M}_{k}$ and $b_{2}=\left(\Lambda_{2}, B_{2}\right) \in \mathscr{M}_{l}$ be two instantons. Then $b_{1}$ and $b_{2}$ correspond through the ADHM construction to based maps $f_{1}: S^{4} \rightarrow$ $G_{n, n+k}(H) \subset B \operatorname{Sp}(n)$ and $f_{2}: S^{4} \rightarrow G_{n, n+l}(H) \subset B \operatorname{Sp}(n)$. In affine coordinates on the Grassmannians we may write $f_{1}(x)=\left(\Lambda_{1}\left(B_{1}-x I\right)^{-1}\right)^{*}$ and $f_{2}(x)=\left(\Lambda_{2}\left(B_{2}-x I\right)^{-1}\right)^{*}$ as long as $x$ is not an eigenvalue of $B$. Since the values of $f_{1}$ and $f_{2}$ at the eigenvalues of $B_{1}$ and $B_{2}$ respectively are well defined by continuity we will abuse the notation and use the matrix equations to globally represent $f_{1}$ and $f_{2}$. Thus we have

$$
\left(f_{1} * f_{2}\right)(x)= \begin{cases}\left(\Lambda_{1}\left(B_{1}-\left(\ln x_{0}+x_{1} i+x_{2} j+x_{3} k\right) I\right)^{-1}\right)^{*} & \text { if } x_{0} \geq 0 \\ \left(\Lambda_{2}\left(B_{2}-\left(\ln \left(-1 / x_{0}\right)+x_{1} i+x_{2} j+x_{3} k\right) I\right)^{-1}\right)^{*} & \text { if } x_{0} \leq 0 .\end{cases}
$$

On the other hand $b_{1} * b_{2}=b_{\delta}=\left(\Lambda_{\delta}, B_{\delta}\right) \in \mathscr{B}_{k+l}^{\varepsilon}$ is represented by the based map $f_{3}: S^{4} \rightarrow G_{n, n+k+l}(H) \subset B \operatorname{Sp}(n)$ given by $f_{3}(x)=\left(\Lambda_{\delta}\left(B_{\delta}-x I\right)^{-1}\right)^{*}$. Of course $f_{3}$ is homotopic to $f_{1} * f_{2}$ as they represent maps from $S^{4}$ into $B \operatorname{Sp}(n)$ of the same degree. However to show that the upper square commutes we must show much more, namely that the homotopy we choose from $f_{3}$ to $f_{1} * f_{2}$ depends continuously on the values of the $b_{i}$. We proceed as follows.

The eigenvalues of $B_{\delta}$ are centered about \pm 1 (this is independent of the choices of $B_{1}$ and $B_{2}$, rather it depends only on the fact that $\delta<\delta_{0}$ ) and thus the eigenvalues of $B_{\delta}$ are disjoint from the $x_{0}=0$ hyperplane in $H$. Hence $\left.f_{3}\right|_{x_{0}=0}$ is contractible in $B \operatorname{Sp}(n)$ and thus there is a canonical homotopy of $f_{3}$ to a map $f_{4}$ defined as follows:

$$
f_{4}(x)= \begin{cases}f_{3}\left(x_{0}-\frac{1}{10}+x_{1} i+x_{2} j+x_{3} k\right) & \text { if } x_{0} \geq \frac{1}{10} \\ \frac{\left|x_{0}\right|}{10} f_{3}\left(x_{1} i+x_{2} j+x_{3} k\right)+\left(1-10\left(\left|x_{0}\right|\right)\right) P & \text { if }\left|x_{0}\right| \leq \frac{1}{10} \\ f_{3}\left(x_{0}+\frac{1}{10}+x_{1} i+x_{2} j+x_{3} k\right) & \text { if } x_{0} \leq \frac{-1}{10}\end{cases}
$$

where $P$ is the $n$-plane representing the base point in $G_{n, n+k+l}(H)$ and the "sum" of two $n$-planes in $G_{n, n+k+l}(H)$ is defined by entry-wise addition of affine coordinates. The formula for $f_{4}$ is well defined as $x$ is never an eigenvalue for $b_{\delta}$ as long as $\left|x_{0}\right|<\frac{1}{10}$. Notice that $f_{4}$ sends the $x_{0}=0$ hyperplane to $P$, the base point in $B \mathrm{Sp}(n)$, and hence $f_{4}$ is canonically a loop sum. Furthermore writing $f_{4}(x)$ in terms of affine coordinates on $G_{n, n+k+l}(H)$ we see that $f_{4}$ is canonically homotopic to

$$
f_{5} * f_{6}= \begin{cases}P_{k} & \text { if } x_{0} \geq 0 \\ P_{l} & \text { if } x_{0} \leq 0\end{cases}
$$


Here $P_{k}$ is an $n$-plane in $H^{n+k+l}$ expressible in terms of the first $n+k$ coordinates (that is, the last $l$ coordinates are zero) and $P_{l}$ is an $n$-plane in $H^{n+k+l}$ expressible in terms of the first $n$ and last $l$ coordinates (that is, the $n+1$ through $n+k$ coordinates are zero). In addition there is a self map of $B \operatorname{Sp}(n)$ which is homotopic to the identity and which interchanges affine coordinates on the Grassmannian. Hence $f_{4}$ is canonically homotopic to

$$
f_{5} * f_{7}= \begin{cases}P_{k} & \text { if } x_{0} \geq 0 \\ P_{l}^{\prime} & \text { if } x_{0} \leq 0\end{cases}
$$

where $P_{l}^{\prime}$ is obtained from $P_{l}$ via the self map of $G_{n, n+k+l}(H)$ induced by the $\operatorname{map}(x, y, z) \mapsto(x, z, y)$ on $H^{n+k+l} \cong H^{n} \times H^{k} \times H^{l}$.

Lastly, as

$$
\left(\Lambda_{j}\left(B_{j}-x I\right)^{-1}\right)^{*}=\left(\frac{\delta}{\left\|b_{j}\right\|} \Lambda_{j}\left(\frac{\delta}{\left\|b_{j}\right\|} B_{j}-\left(\frac{\delta}{\left\|b_{j}\right\|} x\right) I\right)^{-1}\right)^{*} \quad \text { for } j=1,2
$$

we see there is a homotopy $\nu$ of $H$ such that after precomposing by $\nu, f_{1} *$ $f_{2}=f_{5} * f_{7}$. As every homotopy described in the argument above depends continuously on $b_{1}$ and $b_{2}$ the upper square in (6.4) commutes up to homotopy.

The lower square: Let $p=\left(p_{1}, \cdots, p_{k}\right) \in C_{k}\left(R^{4}\right)$ and $q=\left(q_{1}, \cdots, q_{l}\right) \in$ $C_{l}\left(R^{4}\right)$. Then $p * q=\left(1+\delta p_{1}, \cdots, 1+\delta p_{k},-1+\delta q_{1}, \cdots,-1+\delta q_{l}\right) \in C_{k+l}\left(R^{4}\right)$ for some $\delta, 0<\delta<\delta_{0}$. Thus the lower square fails to commute only by a multiple of $\delta$ on the $\Lambda$ matrices (the $B$ matrices are identical). But there is an obvious homotopy on $\mathscr{M}_{k+l}$ using the multiple of $\delta$ on the $\Lambda$ matrix which completes the proof.

The following theorem follows directly from the arguments and constructions used in the proof of Theorem 6.3.

Theorem 6.10. The following diagram commutes up to homotopy:

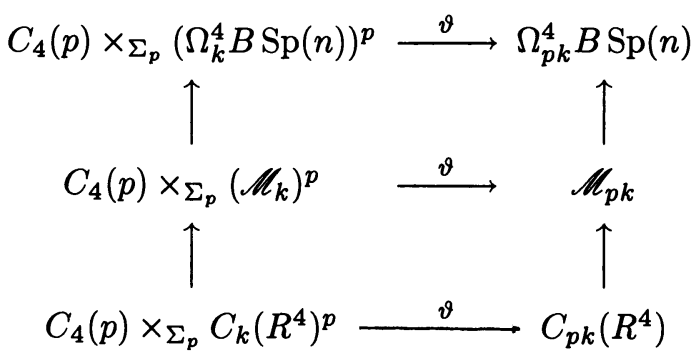

Theorems 6.3 and 6.10 are sufficient to carry out our computational program in $\S \S 9$ and 10 . We close this section with a theorem and general remark, which although not necessary for computational purposes, are interesting in their own right. 
Theorem 6.12. The loop sum maps $*: \mathscr{M}_{k} \times \mathscr{M}_{l} \rightarrow \mathscr{M}_{k+l}$ are homotopy associative and hence $\mathscr{M}$ is a homotopy associative topological monoid.

Proof. We must show that

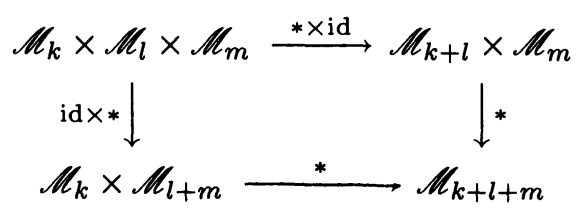

commutes up to homotopy. But both compositions are homotopic to the triple sum $\mathscr{M}_{k} \times \mathscr{M}_{l} \times \mathscr{M}_{m} \rightarrow \mathscr{M}_{k+l+m}$ defined by precomposing the Taubes retraction with the map that sends $\left(b_{1}, b_{2}, b_{3}\right)$ to

$$
B=\operatorname{diag}\left(I+\delta B_{1}, \delta B_{2},-I+\delta B_{3}\right)
$$

and

$$
\Lambda=\left(\delta \Lambda_{1}, \delta \Lambda_{2}, \delta \Lambda_{3}\right)
$$

for all sufficiently small $\delta>0$ via the following homotopy:

$$
B_{t}=\operatorname{diag}\left(\begin{array}{c}
\left(I+(1-t) \delta_{t} I+\delta_{1} \delta_{2} B_{1}\right) \\
(1-t)\left(I-\delta_{2} I\right)+\delta_{1} \delta_{2} B_{2} \\
-I+\delta_{1} \delta_{2} t+(1-t) \delta_{2} B_{3}
\end{array}\right)
$$

and

$$
\Lambda_{t}=\left(\delta_{1} \delta_{2} \Lambda_{1}, \delta_{1} \delta_{2} \Lambda_{2},(1-t) \delta_{2} \Lambda_{3}\right)
$$

q.e.d.

If the loop sum were associative (which it is not) and not just homotopy associative then $\mathscr{M}$ would automatically have a classifying space and $\Omega B(\mathscr{M})$ would be a group completion. Stasheff's theory of $A_{\infty}$ spaces [46] supplies a recognition principle for determining if a homotopy associative monoid does have a classifying space. We do not pursue this question here.

\section{Instanton number one}

We now return to the case $G=\operatorname{Sp}(1)$. In this section we examine the natural inclusion $i_{1}: \mathscr{M}_{1} \rightarrow \mathscr{C}_{1}$ and see that, up to homotopy, $i_{1}$ is equivalent to the well-known $J$-homomorphism $J: \mathrm{SO}(3) \rightarrow \Omega_{1}^{3} S^{3}$. This analysis, while rather straightforward, is a vital ingredient, especially at the primes 2 and 3 , in our homological calculations given in $\S \S 9$ and 10 .

We begin by recalling that $\mathscr{M}_{1}$ is homeomorphic to $\mathrm{SO}(3) \times B^{5}$ [6], and in fact, $\mathscr{M}_{1}$ is the total space of a principal $\mathrm{SO}(3)$ bundle over $\mathscr{M}^{\prime}$, which is 
homeomorphic to $B^{5}$. Furthermore the following commutative diagram is a pullback of principal $\mathrm{SO}(3)$ bundles (see [33]):

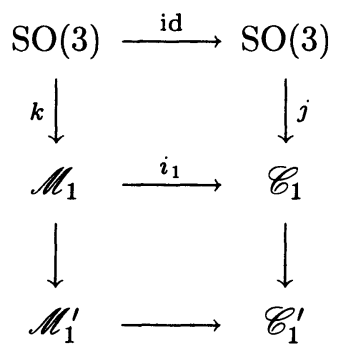

Recall $\mathscr{M}_{1}$ and $\mathscr{C}_{1}$ are the moduli spaces of instantons and connections under the based gauge group, while $\mathscr{M}_{1}^{\prime}$ and $\mathscr{C}_{1}^{\prime}$ are the respective moduli spaces under the full gauge group. In addition we have denoted the natural inclusions of the fibers $\mathrm{SO}(3)$ over the preferred base points by $k$ and $j$ respectively. As $k: \mathrm{SO}(3) \rightarrow \mathscr{M}_{1} \simeq \mathrm{SO}(3) \times B^{5}$ is a homotopy equivalence, the commutativity of the upper square of (7.1) implies that up to homotopy the natural inclusion $i_{1}: \mathscr{M}_{1} \rightarrow \mathscr{C}_{1}$ is equivalent to the inclusion $j: \mathrm{SO}(3) \rightarrow \mathscr{C}_{1}$. Thus, as $\mathscr{C}_{1}$ is homotopy equivalent to $\Omega_{1}^{3} S^{3}$ (let $\theta: \mathscr{C}_{1} \rightarrow \Omega_{1}^{3} S^{3}$ be the equivalence given by Atiyah and Jones [7]), $i_{1}: \mathscr{M}_{1} \rightarrow \mathscr{C}_{1}$ is homotopy equivalent to a map $j^{\prime}=\theta \circ j: \mathrm{SO}(3) \rightarrow \Omega_{1}^{3} S^{3}$.

One of the most important maps in homotopy theory is the $J$ homomorphism, $J: \mathrm{SO}(n) \rightarrow \Omega_{1}^{n} S^{n}$ (see [57] for example). We briefly recall the construction of $J$ for $n=3$ and then show our map $j^{\prime}$ is homotopic to $J$.

We may naturally regard $\mathrm{SO}(n)$ as a subspace of $\operatorname{Map}_{1}\left(S^{n-1}, S^{n-1}\right)$ in the obvious way. Now given a map $\alpha: S^{r} \rightarrow \mathrm{SO}(n)$ we form the adjoint of the composition of $\alpha$ with the inclusion into $\operatorname{Map}_{1}\left(S^{n-1}, S^{n-1}\right)$ to obtain $\operatorname{Ad}(\alpha): S^{r} \times S^{n-1} \rightarrow S^{n-1}$. As $\partial\left(D^{r+1} \times S^{n-1}\right)=S^{r} \times S^{n-1}=\partial\left(S^{r} \times D^{n}\right)$ we may extend $\operatorname{Ad}(\alpha)$ to two maps $D^{r+1} \times S^{n-1} \rightarrow D_{+}^{n}$ and $S^{r} \times D^{n} \rightarrow D_{-}^{n}$ by radially extending $\operatorname{Ad}(\alpha)$ over the two discs $D_{+}^{n}$ and $D_{-}^{n}$ in two different ways. Then these two maps glue together to give a map

$$
J(\alpha): S^{r+n}=\left(D^{r+1} \times S^{n-1}\right) \cup\left(S^{r} \times D^{n}\right) \rightarrow D_{+}^{n} \cup D_{-}^{n}=S^{n}
$$

or equivalently $\operatorname{Ad}(J(\alpha)): S^{r} \rightarrow \Omega_{1}^{n} S^{n}$. The assignment $\alpha \mapsto \operatorname{Ad}\left(J_{\alpha}\right)$ induces a homomorphism $J: \pi_{r}(\mathrm{SO}(n)) \rightarrow \pi_{r}\left(\Omega_{1}^{r} S^{n}\right) \simeq \pi_{r+n}\left(S^{n}\right)$ for all $r$ and $n$. If we let $\alpha: S^{3} \rightarrow R P(3) \simeq \mathrm{SO}(3)$ be the canonical double cover, then $J(\alpha)$ gives an explicit description of the map $J: \mathrm{SO}(3) \rightarrow \Omega_{1}^{3} S^{3}$ as follows.

Let $x=x_{0}+x_{1} i+x_{2} j+x_{3} k$ be a unit quaternion. Then $x \in S^{3} \subset H^{1}$ and $S^{2}=\left\{x \in S^{3}: x_{0}=0\right\}$. Let $q \in S^{3}$; then $J(\alpha)$ is the extension of the map $S^{3} \times S^{2} \rightarrow S^{2}$ given by $(q, x) \mapsto q x q^{-1}[47]$. Regarding $S^{6}=D^{4} \times S^{2} \cup S^{3} \times D^{3}$ 
and $S^{3}=D_{+}^{3} \cup D_{-}^{3}$ we have

$$
\begin{gathered}
\left.J(\alpha)\right|_{D^{4} \times S^{2}}(2 t q, x)=\left((\sin \pi t) q x q^{-1}, \cos \pi t\right) \quad \text { for } 0 \leq t \leq \frac{1}{2}, \\
\left.J(\alpha)\right|_{S^{3} \times D^{3}}(q,(2-2 t) x)=\left((\sin \pi t) q x q^{-1}, \cos \pi t\right) \quad \text { for } \frac{1}{2} \leq t \leq 1,
\end{gathered}
$$

where the second coordinate is the suspension coordinate in $S^{3}=D_{+}^{3} \cup D_{-}^{3}$. Notice that $\operatorname{Ad}(J(\alpha))(q)=\operatorname{Ad}(J(\alpha))(-q)$ and thus yields a map from $\mathrm{SO}(3)$ to $\Omega_{1}^{3} S^{3}$, which is precisely $J: \mathrm{SO}(3) \mapsto \Omega_{1}^{3} S^{3}$.

Lemma 7.5. $j^{1}=\theta \circ j: \mathrm{SO}(3) \rightarrow \mathscr{C}_{1} \rightarrow \Omega_{1}^{3} S^{3}$ is homotopic to $J: \mathrm{SO}(3)$ $\rightarrow \Omega_{1}^{3} S^{3}$.

Proof. We begin by recalling the Atiyah-Jones equivalence $\theta$ in more detail. We may first assume that $\mathscr{C}_{1}$ has been deformed to $\mathscr{C}_{1}^{F}$, the moduli space of connections that are flat near infinity. For any connection $\omega \in \mathscr{C}_{1}^{F}$ choose a covariantly constant section $\alpha: S^{4} \rightarrow P_{1}$ with $\alpha(\infty)=p_{\infty}$ and any section $\beta:\left.R^{4} \rightarrow P_{1}\right|_{R^{4}}$ which agrees with $\alpha$ on a fixed radial direction $S_{0}$. On a small $S^{3}$ centered at $\infty, \alpha \beta^{-1}$ defines a map $S^{3} \rightarrow G$. Then the map $\omega \mapsto \alpha \beta^{-1}$ can be shown to be a homotopy equivalence. Now let $q \in S^{3}$ and $f_{q}: S^{3} * S^{3} \rightarrow S^{3} * S^{3}$ be defined by $f_{q}(x, t, y)=(q x, t, q y)$. It is clear that $f_{q}$ is a gauge transformation which fixes the fiber at $\infty$ only when $q=+1$. For $f \in \mathscr{G}\left(P_{1}\right)$ we recall that $f^{*}(\omega)=\operatorname{Ad}_{f}^{*}(\omega)+f^{-1} d f$ and thus, as $d f_{q}=0$, we have $f_{q}^{*}(\omega)=\operatorname{Ad}_{f_{q}}^{*}(w)$. This defines an action $S^{3} \times \mathscr{C}_{1} \rightarrow \mathscr{C}_{1}$ given by $(q, \omega) \mapsto f_{q}^{*}(\omega)$ which passes to the quotient action

$$
\mathrm{SO}(3) \times \mathscr{C}_{1} \rightarrow \mathscr{C}_{1}
$$

given by $(\{q\}, \omega) \mapsto f_{q}^{*}(\omega)$. For a fixed $\omega \in \mathscr{C}_{1}$ the orbit of this action describes the fiber over $\{\omega\}$ in the principle $\mathrm{SO}(3)$ bundle $\mathscr{C}_{1} \rightarrow \mathscr{C}_{1}^{\prime}$.

The action in (7.6) and the homotopy equivalence $\theta$ yield the following commutative diagram of action maps:

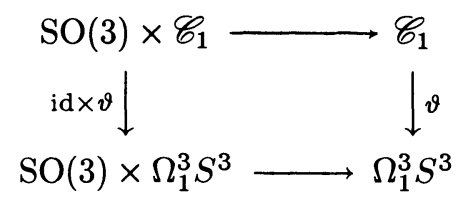

However if we apply the gauge transformation $f_{q}$ to $P_{1}$ we will clearly send the section $\alpha$ to the section $q \alpha$ and the section $\beta$ to the section $q \beta$. Thus if $\theta(w)=\alpha \beta^{-1}$, then $\theta\left(f_{q}^{*}(\omega)\right)=q \alpha \beta^{-1} q^{-1}$ and the bottom horizontal map in (7.7) is given by $(q, f) \mapsto q f q^{-1}$.

If we set $f=\mathrm{id} \in \Omega_{1}^{3} S^{3}$ we find that $j^{\prime}: \mathrm{SO}(3) \rightarrow \Omega_{1}^{3} S^{3}$ has adjoint map $\operatorname{Ad}\left(j^{\prime}\right)=\mathrm{SO}(3) \times S^{3} \rightarrow S^{3}$ given by $(q, y) \mapsto q y q^{-1}$ for $y \in S^{3}$. Now writing $y=t+x$ with $x=y_{1} i+y_{2} j+y_{3} k$ and comparing with formulas (7.3) and (7.4) immediately establishes the lemma. 


\section{Homology operations on $C_{4}$-spaces}

Theorems 6.3 and 6.10 show that $\mathscr{M}$ is a "homotopy $C_{4}$ space" in the sense that $(\mathscr{M}, \vartheta)$ is homotopy compatible with certain important $C_{4}$ operad structure maps on $\amalg C_{k}\left(R^{4}\right)$ and $\Omega^{4} B \operatorname{Sp}(n)$. In this section we recall certain fundamental facts about the homology of $C_{4}$ spaces which we will need in $\S \S 9$ and 10.

The homology theory of iterated loop spaces is very rich and it is well known that $H_{*}\left(\Omega^{n} X, Z / p\right)$ admits homology operations that are not the duals of the Steenrod operations. Araki and Kudo [1] were the first to discover and study such operations, which generalize the Pontrjagin product induced by loop sum, when $p=2$. Browder [14] obtained more complete information when $p=2$ and also studied when $p$ is an odd prime. Dyer and Lashof [24] then studied the algebra of stable operations (on infinite loop spaces) for all primes $p$. These stable operations (which naturally give rise to unstable operations on iterated loop spaces) and their rich algebraic structure have made the Dyer-Lashof algebra a fundamental tool in algebraic topology. However not all unstable homology operations come from stable Dyer-Lashof operations (to quote Fred Cohen [16], "To be precise, only 1/(p-1) times the requisite number of operations (defined in this paper [16]) may be described using the methods of Dyer and Lashof.") and we will consider other homology operations here as well. May's theory of iterated loop spaces [36], [16], shows that all the homology operations mentioned above live naturally on $C_{n}$ operad spaces. Finally Cohen [16] has given a complete treatment of all the $\bmod p$ homology operations on $C_{4}$ spaces that we consider in this paper.

We now recall the definitions of these operations. We do not give all the rules one uses to compute with these operations but refer the reader to Cohen's concise yet encyclopedic treatment [16, §1, pp. 213-219].

Definition 8.1. Let $X$ be a $C_{n+1}$ space with $x \in H_{q}(X, Z / p)$ and $y \in$ $H_{r}(X, Z / p)$. Then define the following.

(a) For $i<n$

$$
Q_{i(p-1)}(x)=\vartheta_{p^{*}}\left(e_{i(p-1)} \otimes x^{p}\right) \in H_{p q+i(p-1)}(X, Z / p)
$$

and, for $p$ odd,

$$
Q_{i(p-1)-1}(x)=\vartheta_{p^{*}}\left(e_{i(p-1)-1} \otimes x^{p}\right) \in H_{p q+i(p-1)-1}(X, Z / p) .
$$

(b) For $p=2$ and $s<q$

$$
Q^{s}(x)=0
$$

while if $s \geq q$ then

$$
Q^{s}(x)=Q_{s-q}(x)
$$


(c) For $p>2$ and $2 s<q$

$$
Q^{s}(x)=0
$$

while if $2 s \geq q$ then

$$
Q^{s}(x)=(-1)^{s} \nu(q) Q_{(2 s-q)(p-1)}(x) .
$$

(d) For $2 s \leq q$

$$
\beta Q^{s}(x)=0
$$

while if $2 s>q$

$$
\beta Q^{s}(x)=(-1)^{s} \nu(q) Q_{(2 s-q)(p-1)-1}(x),
$$

where $\nu(q)=(-1)^{q(q-1)(p-1) / 4}(((p-1) / 2) !)^{q}$.

(e) For $p=2$

$$
\xi_{n}(x)=\vartheta_{2}\left(e_{n} \otimes x \otimes x\right) \in H_{2 q+n}(X, Z / p) .
$$

(f) For $p$ odd

$$
\xi_{n}=(-1)^{(n+q) / 2} \nu(q) \vartheta_{p^{*}}\left(e_{n(p-1)} \otimes x^{p}\right) \in H_{p q+n(p-1)}(X, Z / p) .
$$

(g) For $p$ odd and $n+q$ even

$$
\zeta_{n}=(-1)^{(n+q) / 2} \nu(q) \vartheta_{p^{*}}\left(e_{n(p-1)-1} \otimes x^{p}\right) \in H_{p q+n(p-1)-1}(X, Z / p) .
$$

(h)

$$
\lambda_{n}(x, y)=(-1)^{n q+1} \psi_{*}(\iota \otimes x \otimes y) \in H_{n+q+r}(X, Z / p) .
$$

Here $\psi: C_{n+1}(2) \times X \times X \rightarrow X$ is the $\Sigma_{2}$ equivariant map without the $Z / 2$ quotient action on the domain and $\iota \in H_{n}\left(C_{n+1}(2), Z / p\right) \cong H_{n}\left(S^{n}, Z / p\right)$ is the fundamental class.

Remarks. 1. Part (a) defines the operations which come from the stable Dyer-Lashof operations [24].

2. Parts (b) and (c) provide a dictionary for passing between lower notation $\left(Q_{a}(x)\right)$ and upper notation $\left(Q^{b}(x)\right)$, which was invented by May [35] to simplify many computational formulas involving iterated operations, especially at odd primes. We shall not strictly adhere to one convention but rather pass freely to whichever notation can be used most easily to state our results.

3. Part (h) defines the Browder operation [14].

4. The cells $e_{i} \in H_{i}\left(C_{n}(p) / \Sigma_{p}, Z / p(q)\right)$ are dual to the $i$-dimensional generator in the image of $H^{i}\left(B \Sigma_{p}, Z / p(q)\right) \rightarrow H^{i}\left(C_{n}(p) / \Sigma_{p}, Z / p(q)\right.$ ) (see [16]).

5. $Q_{0}(x)=x^{p}$, the $p$-fold Pontrjagin product of $x$ with itself.

6. In general the top operation $\xi_{n}$ behaves very much like a Dyer-Lashof operation $\left(\xi_{n}=Q_{n(p-1)}\right.$ if $X$ is a $C_{n+2}$ operad space). Theorem 1.3 of [16] catalogs the precise differences.

7. We are interested in $C_{4}$ spaces thus $n=3$ in our calculations. 
We conclude this section with the following classical facts used in $\S \S 9$ and 10 :

Theorem 8.2. (a) $H_{*}(\mathrm{SO}(3), Z / 2) \simeq E\left(x_{1}, x_{2}\right)$, an exterior algebra over $Z / 2$, where $\left|x_{1}\right|=1,\left|x_{2}\right|=2$ and the Pontrjagin product in $H_{*}(\mathrm{SO}(3), Z / 2)$ is induced by the Lie group multiplication on $\mathrm{SO}(3)$.

(b) $H_{*}(\mathrm{SO}(3), Z / p) \simeq E\left(x_{3}\right)$, an exterior algebra over $Z / p$ on a single generator of dimension 3 . Here $p$ is an odd prime.

To describe $H_{*}\left(\Omega^{m+1} S^{m+1}, Z / p\right)$ recall the identity map $S^{m+1} \rightarrow S^{m+1}$ represents the base point in the 1 component $\Omega_{1}^{m+1} S^{m+1}$ and thus a distinguished homology class [1] $\in H_{0}\left(\Omega_{1}^{m+1} S^{m+1}, Z / p\right)$. Furthermore if $x$ and $y$ are homology classes carried by the $k$ and $l$ components of $\Omega^{m+1} S^{m+1}$, then $x * y$ and $Q_{i}(x)$ are carried by the $k+l$ and $p k$ components respectively.

Theorem 8.3 [24]. $\quad H_{*}\left(\Omega^{m+1} S^{m+1}, Z / 2\right) \simeq Z / 2\left([1], Q_{I}(1)\right)$, a polynomial algebra over $Z / 2$, under the loop sum Pontrjagin product, on generators [1] and $Q_{I}(1)=Q_{i_{1}} Q_{i_{2}} \cdots Q_{i_{k}}(1)$, where $I=\left(i_{1}, \cdots, i_{k}\right)$ satisfies $0<i_{1} \leq i_{2} \leq \cdots \leq i_{k} \leq m$.

As is customary we have written $Q_{m}$ for $\xi_{m}$. Notice that $H_{*}\left(\Omega^{3} S^{3}, Z / 2\right)$ can be described solely in terms of iterated operations $Q_{0}, Q_{1}$ and $Q_{2}$. However, as $\Omega^{3} S^{3} \simeq \Omega^{4} B S^{3}, Q_{3}=\xi_{3}$ exists in $H_{*}\left(\Omega^{4} B S^{3}, Z / 2\right)$ and in $H_{*}(\mathscr{M}, Z / 2)$ where it is highly nontrivial as we shall see in $\S 9$. To state the analog of 8.3 for odd primes we need a bit more notation. Let $\beta^{\varepsilon_{1}} Q^{s_{1}} \cdots \beta^{\varepsilon_{k}} Q^{s_{k}}(1)=Q^{I}(1)$ be an iterated $\bmod p$ operation on [1].

Definition 8.4 [16]. Let $I=\left(\varepsilon_{1}, s_{1}, \cdots, \varepsilon_{k}, s_{k}\right)$ and $p$ be an odd prime.

(A) $e(I)=2 s_{1}-\varepsilon_{1}-\sum_{j=2}^{k}\left(2 s_{j}(p-1)-\varepsilon_{j}\right)$ is the excess of $I$.

(B) $I$ is admissible if $p s_{j}-\varepsilon_{j} \geq s_{j-1}$ for $2 \leq j \leq k$.

(C) $b(I)=\varepsilon$.

Theorem 8.5 [16]. Let $p$ be an odd prime. As algebras, under the loop sum Pontrjagin product:

(a) $H_{*}\left(\Omega^{4} B S^{3}, Z / p\right) \cong H_{*}\left(\Omega^{3} S^{3}, Z / p\right) \simeq S\left([1], Q^{I}(1)\right)$, where $I$ is admissible, $\varepsilon(I)+b(I)>0, s_{k} \leq 1$.

(b) $H_{*}\left(\Omega^{4} S^{4}, Z / p\right) \cong \bar{S}\left([1], Q^{I}(1), Q^{J}\left(\lambda_{3}(1,1)\right)\right)$, where $I, J$ are admissible, $\varepsilon(I)+b(I)>0, e(J) \geq 3, s_{k}(I) \leq 1, s_{k}(J) \leq 3$.

Remarks. 1. $S($ ) is the tensor product over $Z / p$ of polynomial algebras on even dimensional generators and of exterior algebras on odd dimensional generators.

2. The Browder operation $\lambda_{3}(1,1)$ is nontrivial and indecomposable in terms of the $Q^{I}$ 's in $H_{*}\left(\Omega^{4} S^{4}, Z / p\right)$ and hence in $H_{*}\left(\amalg C_{k}\left(R^{4}\right), Z / p\right)$. However, $\lambda_{3}(1,1)$ is zero in $H_{*}\left(\Omega^{4} B S^{3}, Z / p\right)$. For $p \geq 5$ this fact follows immediately from dimensional considerations whereas for $p=3$ it requires proof. We thank Fred Cohen for communicating a proof to us. 
3. $Q_{0}, Q_{p-1}, Q_{2(p-1)}, Q_{3(p-1)}=\xi_{3}$ and their Bockstein's are all defined in $H_{*}\left(\Omega^{4} B S^{3}, Z / p\right)$ and $H_{*}\left(\Omega^{4} S^{4}, Z / p\right)$ although for any fixed homology class $x$ only $Q_{0}(x), Q_{2(p-1)}(x)$ or $Q_{(p-1)}(x), Q_{3(p-1)}(x)$ are defined (depending on the parity of $|x|$ as $0 \leq 2 s-|x| \leq 3)$.

Theorem 8.6. Let $J: \mathrm{SO}(3) \rightarrow \Omega_{1}^{3} S^{3}$ be the classical J-homomorphism. Then $J_{*}: H_{*}(\mathrm{SO}(3), Z / p) \rightarrow H_{*}\left(\Omega_{1}^{3} S^{3}, Z / p\right)$ is given by the following formulas:

(A) If $p=2$,

$$
\begin{aligned}
& J_{*}\left(x_{1}\right)=Q_{1}(1) *[-1], \\
& J_{*}\left(x_{2}\right)=Q_{2}(1) *[-1], \\
& J_{*\left(x_{1} x_{2}\right)}=Q_{2}(1) * Q_{1}(1) *[-3]+Q_{1} Q_{1}(1) *[-3]+\left(Q_{1}(1)\right)^{3} *[-5] .
\end{aligned}
$$

(B) If $p=3$,

$$
J_{*}\left(x_{3}\right)=Q_{3}(1) *[-2]=-\beta Q^{I}(1) *[-2] .
$$

(C) If $p \geq 5$,

$$
J_{*} \equiv 0 .
$$

Proof. (A1), (A2), (B) and (C) are well known (see [24], [39], [16], or [34] for example). (A3) follows from the commutative diagram,

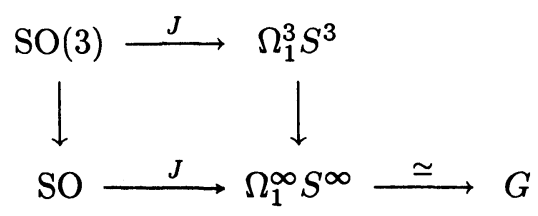

the fact that the Pontrjagin product in $\mathrm{SO}(3)$ is compatible with the composition product $x \circ y$ in $\Omega_{1}^{\infty} S^{\infty} \sim G$, and Milgram's distributivity formula [39], that permits the calculation of $\left(Q_{2}(1) *[-1]\right) \circ\left(Q_{1}(1) *[-1]\right)$ in terms of the loop sum. q.e.d.

We close this section with three immediate corollaries of Lemma 7.5 and Theorem 8.6.

Corollary 8.8. $H_{q}\left(\mathscr{M}_{1}, Z / 2\right) \cong Z / 2$ if $0 \leq q \leq 3$ and 0 if $q>3$. For $p$ an odd prime, $H_{q}\left(\mathscr{M}_{1}, Z / p\right) \cong Z / p$ if $q=0$ or 3 and 0 if $q \neq 0$ or 3 .

Corollary 8.9. The natural inclusion $i: \mathscr{M}_{1} \rightarrow \mathscr{C}_{1}$ induces a monomorphism in $Z / 2$ and $Z / 3$ homology and is trivial in $Z / p$ homology for $p \geq 5$. Furthermore, if we write $x_{3}$ for $x_{1} x_{2} \in H_{3}(\mathrm{SO}(3), Z / 2)$, and $z_{q}$ for the $q$-dimensional generator of $H_{*}\left(\mathscr{M}_{1}\right)$, then $i_{*}\left(z_{q}\right)=J\left(x_{q}\right)$ for all $q$ and primes $p$. 
Corollary 8.10. The natural inclusion $i: \mathscr{M}_{1} \rightarrow \mathscr{C}_{1}$ induces an isomorphism $H$ for $q=1$ and an injection for $q=2$ whereas $i_{*}: H_{3}\left(\mathscr{M}_{1}, Z\right) \rightarrow$ $H_{3}\left(\mathscr{C}_{1}, Z\right)$ is isomorphic to the natural epimorphism of $Z$ onto a $Z / 12$ factor. Hence $\operatorname{ker}\left(i_{1}\right)_{3}$ is isomorphic to $Z$.

\section{Mod two calculations}

We are now ready to use the results of the previous sections to construct many new nontrivial classes in $H_{*}\left(\mathscr{M}_{k}, Z / 2\right)$. Theorems 6.3 and 6.10 imply that the following diagrams commute:

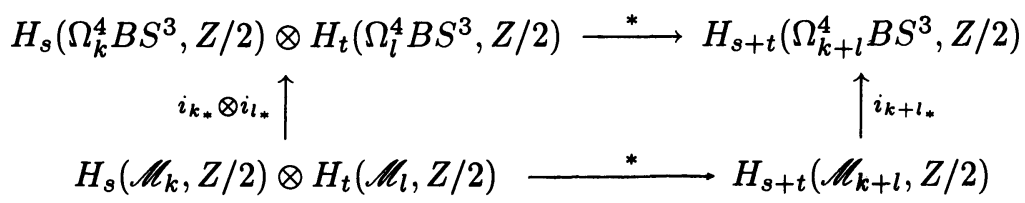

and, for $i \leq 3$ :

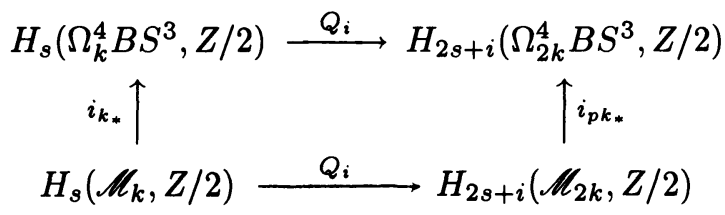

We may use our structure maps $\vartheta_{j}: C_{4}(j) \times_{\Sigma_{j}} \mathscr{M}_{k}^{j} \rightarrow \mathscr{M}_{j k}$ to define classes in $H_{*}(\mathscr{M})$ using the formulas given in Definition 8.1. These diagrams imply that the images of our classes in $H_{*}\left(\Omega^{4} B S^{3}\right)$ are fully compatible with the usual $C_{4}$ homology operations in $\Omega^{4} B S^{3}$. Thus by abuse of notation we will use the notation of Definition 8.1 to designate our homology operations in $H_{*}(\mathscr{M})$.

If we start with the generator [1] $\in H_{0}\left(\mathscr{M}_{1}, Z / 2\right)$ and compute iterated Dyer-Lashof operations on [1], and loop sums of such elements by using the commutativity of diagrams (9.1) and (9.2) and the known loop sum Dyer-Lashof structure of $H_{*}\left(\Omega^{4} B S^{3}, Z / 2\right)$ we may recover the $Z / 2$ version of the Atiyah-Jones theorem that $H_{q}\left(\mathscr{K}_{k}\right) \rightarrow H_{q}\left(\mathscr{C}_{k}\right)$ is a surjection for $k \gg q$. For example we immediately find $Q_{1}(1)$ and $Q_{2}(1)$ are in the image $i_{*}: H_{*}\left(\mathscr{M}_{2}, Z / 2\right) \rightarrow H_{*}\left(\mathscr{C}_{2}, Z / 2\right)$ and, by induction, easily obtain a preliminary result.

Proposition 9.3. Let $I_{k}=\left(i_{1}, \cdots, i_{l}\right)$ with $0 \leq i_{1} \leq \cdots \leq i_{l} \leq 2$. Then

(A) $Q_{I_{l}}(1) \in H_{*}\left(\mathscr{C}_{2^{l}}, Z / 2\right)$ is in the image of $i_{*}$.

(B) $Q_{I_{l_{1}}}(1) * Q_{I_{l_{2}}}(1) * \cdots * Q_{I_{l_{j}}}(1) \in H_{*}\left(\mathscr{C}_{k}, Z / 2\right)$ is in the image of $i_{*}$ for $k \geq \sum_{n=1}^{j} 2^{l_{j}}$. 
We note in passing that $Q_{2} Q_{2} \cdots Q_{2}(1) \in H_{2^{l+1}-2}\left(\mathscr{C}_{2^{l}}, Z / 2\right)$ is in image $\left(i_{*}\right)$ and thus we obtain a nonzero class in $H_{q}\left(\mathscr{M}_{k}, Z / 2\right)$ for $q$ approximately $\frac{1}{4} \operatorname{dim}\left(\mathscr{M}_{k}\right)$. Furthermore, using these elements it is possible to obtain a bound for the range $q=q(k)$ through which $i_{*}$ is a surjection.

Corollary 9.4. With $Z / 2$ coefficients, $i_{*}$ is a surjection for $q \leq k$.

Proof. By induction on $k$. The case $k=1$ follows from Lemma 7.5. The inductive step follows easily from the known structure of $H_{*}\left(\Omega^{4} B S^{3}, Z / 2\right)$ and 9.3. q.e.d.

It is apparent that this method does not give a nontrivial class in ker $i_{*}$ for $q \leq k$ which is consistent with the Atiyah-Jones conjecture. The reader will have undoubtably noticed that we have not yet used the top operation $Q_{3}$ nor Corollary 8.8 in our computations. We shall now see that we can greatly improve Proposition 9.3 by incorporating these results. However, Corollary 9.4 cannot be improved by our methods, rather our computations suggest $i_{*}$ may be neither a surjection nor an injection for $q>k$. Of course neither phenomena is inconsistent with the truth of the Atiyah-Jones conjecture (for $q \leq k)$. We make these statements more precise in what follows.

Starting with the generators $z_{i} \in H_{i}\left(\mathscr{M}_{1}, Z / 2\right)$ for $i=1,2,3$ we may construct elements $Q_{I}\left(z_{i}\right) \in H_{*}\left(\mathscr{M}_{2^{k}}, Z / 2\right)$ (with $i_{k} \leq 3$ ) and recast 9.3. In the appendix we have written out $H_{*}\left(\mathscr{M}_{k}, Z / 2\right)$ for small $k$ detected in this way and are content here to give the following general results:

Proposition 9.5. $z_{i} *[1]=Q_{i}(1) \in H_{i}\left(\mathscr{M}_{2}, Z / 2\right)$ for $i=1,2,3$.

Proof. $z_{i} *[1]$ is detected by the map SO(3) $\rightarrow \mathscr{M}_{1} \times 1 \rightarrow \mathscr{M}_{1} \times \mathscr{M}_{1} \stackrel{*}{\rightarrow} \mathscr{M}_{2}$ while $Q_{i}(1)$ is detected by the map $\mathrm{SO}(3) \rightarrow S^{3} \times_{Z / 2} 1 \times 1 \rightarrow S^{3} \times_{Z / 2} \mathscr{M}_{1} \times$ $\mathscr{M}_{1} \stackrel{\vartheta}{\rightarrow} \mathscr{M}_{2}$. Since $\pi_{1}\left(\mathscr{M}_{2}\right) \cong H_{1}\left(\mathscr{M}_{2}, Z / 2\right) \cong Z / 2$ [28], and $H^{*}(\mathrm{SO}(3), Z / 2)$ is a truncated polynomial algebra on the 1-dimensional generator, the proposition follows from the fact that the two detecting maps have the same effect on $H_{1}$. But $i_{*}\left(z_{1} *[1]\right)=Q_{1}(1)=i_{*}\left(Q_{1}(1)\right)$. q.e.d.

Proposition 9.5 has the following amusing corollary.

Corollary 9.6. $Q_{3}(1)=Q_{1} Q_{1}(1) *[-2]+Q_{2}(1) * Q_{1}(1) *[-2]+Q_{1}(1)^{3} *$ [-4] in $H_{3}\left(\Omega_{2}^{4} B S^{3}, Z / 2\right) \simeq H_{3}\left(\Omega_{2}^{3} S^{3}, Z / 2\right)$.

We now adopt the conventions $z_{0}=[1]$ and $Q_{\phi}\left(z_{i}\right)=z_{i}$ for all $i$. Then we have

Theorem 9.7. $\quad H_{*}\left(\mathscr{M}_{k}, Z / 2\right)$ contains elements of the form

$$
z=z\left(I_{1}, \cdots, I_{n}, j_{1}, \cdots, j_{n}\right)=Q_{I_{1}}\left(z_{j_{1}}\right) * \cdots * Q_{I_{n}}\left(z_{j_{n}}\right)
$$


for all sequences $\left(I_{1}, \cdots, I_{n}, j_{1}, \cdots, j_{n}\right)$ such that $\sum_{m=1}^{n} 2^{l\left(I_{m}\right)} \leq k$. Here each $I_{m}=\left(i_{1}, \cdots, i_{l\left(I_{m}\right)}\right)$ is an admissible sequence $0 \leq i_{1} \leq \cdots \leq i_{l\left(I_{m}\right)} \leq 3$ and $0 \leq j_{a} \leq 3$ for all $1 \leq a \leq n$. Furthermore the image of $z$ in $H_{*}\left(\mathscr{C}_{k}, Z / 2\right)$ is given by replacing $z_{1}, z_{2}$ and $z_{3}$ in 9.5 by $Q_{1}(1) *[-1], Q_{2}(1) *[-1]$, and $\left(Q_{1} Q_{1}(1) *[-3]+Q_{2}(1) * Q_{1}(1) *[-3]+\left(Q_{1}(1)\right)^{3} *[-5]\right)$ respectively.

Corollary 9.9. Let $k=2^{j}$. Then $Q_{3} Q_{3} \cdots Q_{3}\left(z_{3}\right) \in H_{6 k-3}\left(\mathscr{M}_{k}, Z / 2\right)$ has nonzero image in $H_{6 k-3}\left(\mathscr{C}_{k}, Z / 2\right)$.

Proof. Direct computation, using the Adem relations and the weight filtration [16], shows $i_{*}\left(Q_{3} \cdots Q_{3}\left(z_{3}\right)\right)$ may be expressed as

$$
Q_{2} \cdots Q_{2}(1) * Q_{1} \cdots Q_{1}(1) * Q_{0} \cdots Q_{0}(-3)+\text { "terms of higher weight" }
$$

and is thus nonzero in $H_{6 k-3}\left(\mathscr{C}_{k}, Z / 2\right)$. q.e.d.

Theorem 9.7 and Corollary 9.9 point out the existence of nonzero classes in image $i_{*}$ of dimension up to approximately $\frac{3}{4} \operatorname{dim}\left(\mathscr{M}_{k}\right)$. This should be contrasted with the remark following Proposition 9.3. We are able to see this additional homology for the following two reasons. First, as $\mathscr{C}_{k} \simeq \Omega_{k}^{3} S^{3}$, its homology can be completely described (at least additively) by the $Q_{i}$ 's for $i \leq 2$. However $\Omega^{3} S^{3} \simeq \Omega^{4} B S^{3}$ and the operation $Q_{3}$, while decomposable in $\Omega^{3} S^{3}$ and $\mathscr{M}$, is definitely not zero there. We would like to thank Fred Cohen for forcefully pointing out this critical fact to us. Second, rather than just operating on [1] we have used Lemma 7.5 and Corollary 8.8 in a fundamental way. Close examination of 9.3 and 9.7 will show how pervasive an effect these observations have on our knowledge of $H_{*}\left(\mathscr{M}_{k}, Z / 2\right)$.

Returning to Corollary 9.4 it is natural to ask if the bound $q=q(k) \leq k$ through which $i_{*}$ is a surjection can be improved. The answer is no (at least using the methods developed in this paper), for example $\left(Q_{1}(1)\right)^{k+1} *[-k-2] \epsilon$ $H_{k+1}\left(\mathscr{C}_{k}, Z / 2\right)$ cannot be shown to be in the image of $i_{*}$ for $k \leq 4$ using Theorem 9.7 (and we conjecture the restriction $k<4$ can be dropped). Of course since $\mathscr{M}_{k}$ is finite dimensional and $\mathscr{C}_{k}$ has homology in every dimension, surjectivity must fail sooner or later. One of the reasons it apparently fails sooner $(q=k+1)$ rather than later $(q>k+1)$ is the decomposability of $Q_{3}$ in $\Omega^{3} S^{3}$ which also forces candidates for ker $i_{*}$ (for $q>k$ ) as follows.

Proposition 9.10. $Q_{1}\left(z_{1}\right)+z_{2} * z_{1}+z_{3} *[1]$ and $Q_{2}\left(z_{1}\right)+z_{3} * z_{1}$ are in the kernel of $i_{*}: H_{*}\left(\mathscr{M}_{2}, Z / 2\right) \rightarrow H_{*}\left(\mathscr{C}_{2}, Z / 2\right)$.

Proof. The proof follows from direct computation using 9.7 and the standard Dyer-Lashof formulas [16].

Many other examples can easily be constructed, some appear in the appendices. It is not apparent that the classes given in 9.10 are zero in $H_{*}\left(\mathscr{M}_{2}, Z / 2\right)$; however, we have not yet been able to show they are, in fact, nontrivial. We summarize these questions left open by our analysis in $\S 11$. 


\section{Calculations at odd primes}

We now turn our attention to $H_{*}\left(\mathscr{M}_{k}, Z / p\right)$ for $p$ an odd prime. As the proofs of the results of this section are so similar in spirit to the proofs when $p=2$ we simply state our results leaving detailed verification to aficionados of the Dyer-Lashof algebra at odd primes. Again Theorems 6.3 and 6.10 imply the following diagrams commute:

$$
\begin{gathered}
H_{r}\left(\Omega_{k}^{4} B S^{3}, Z / p\right) \otimes H_{t}\left(\Omega_{l}^{4} B S^{3}, Z / p\right) \stackrel{*}{\longrightarrow} H_{r+t}\left(\Omega_{k+l}^{4} B S^{3}, Z / p\right) \\
i_{k_{*}} \otimes i_{l_{*}} \uparrow \\
H_{r}\left(\mathscr{M}_{k}, Z / p\right) \otimes H_{t}\left(\mathscr{M}_{l}, Z / p\right) \stackrel{i_{k+l_{*}}}{\longrightarrow} H_{r+t}\left(\mathscr{M}_{k+l}, Z / p\right) ; \\
H_{r}\left(\Omega_{k}^{4} B S^{3}, Z / p\right) \stackrel{Q^{s}}{\longrightarrow} H_{2 s(p-1)+r}\left(\Omega_{p k}^{4} B S^{3}, Z / p\right) \\
i_{k_{*} \uparrow} \uparrow_{i_{p k_{*}}}^{\longrightarrow} H_{2 s(p-1)+r}\left(\mathscr{M}_{p k}, Z / p\right) ; \\
H_{r}\left(\mathscr{M}_{k}, Z / p\right) \stackrel{Q^{s}}{\longrightarrow} H_{r+t+3}\left(\Omega_{k+l}^{4} B S^{3}, Z / p\right) \\
H_{r}\left(\Omega_{k}^{4} B S^{3}, Z / p\right) \otimes H_{t}\left(\Omega_{l}^{4} B S^{3}, Z / p\right) \stackrel{\lambda_{3}}{\longrightarrow} H_{i_{k+l *}} \\
i_{k_{*}} \otimes i_{l_{*}} \uparrow
\end{gathered}
$$
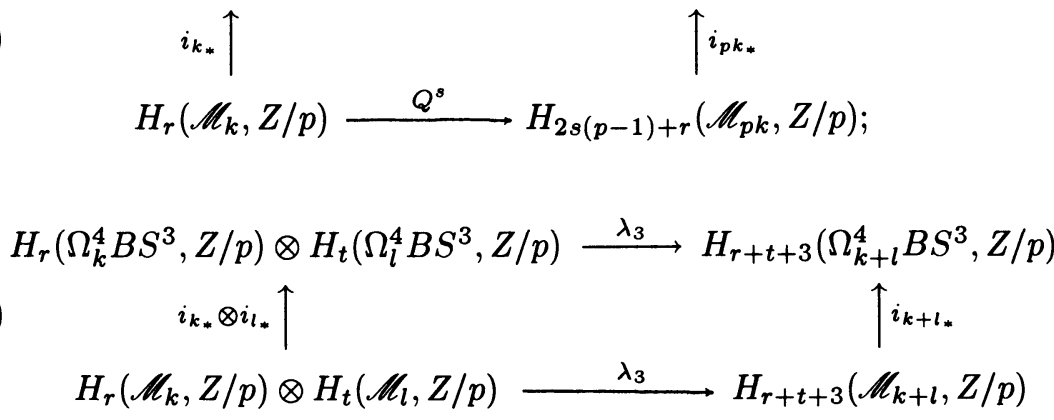

where the last diagram corresponds to the Browder operation $\lambda_{3}$. As in $\S 9$ we may start with [1] $\in H_{0}\left(\mathscr{M}_{1}, Z / p\right)$ and directly obtain the analogs of 9.3 , 9.4 and 9.5.

Proposition 10.4. Let $I$ be an admissible sequence of length $l$ with $\varepsilon(I)+$ $b(I)>0$ and $s_{l}=1$. Then

(A) $Q^{I}(1) \in H_{*}\left(\mathscr{C}_{p^{l}}, Z / p\right)$ is in the image of $i_{*}$,

(B) $Q^{I_{1}}(1) * Q^{I_{2}}(1) * \cdots * Q^{I_{j}}(1) \in H_{*}\left(\mathscr{C}_{k}, Z / p\right)$ is in the image of $i_{*}$ for $k \geq \sum_{n=1}^{j} p^{l\left(I_{j}\right)}$.

Corollary 10.5. With $Z / p$ coefficients, $i_{*}$ is a surjection for $q \leq k$.

We note that, for many choices of $k$ and $p, q$ can be chosen to be greater than $k$ in 10.5. For example if $p \leq k<2 p$ then $i_{*}$ is a surjection for $q<4 p-5$.

Proposition 10.6. $z_{3} *[2]=-\beta Q^{1}(1) \in H_{3}\left(\mathscr{M}_{3}, Z / 3\right)$.

The analog of 9.7 splits into two cases, $p \geq 5$ and $p=3$. Since the $J$ homomorphism, $J: \mathrm{SO}(3) \rightarrow \Omega_{1}^{3} S^{3}$, is trivial in $Z / p$ homology if $p \geq 5$ we cannot improve Proposition 10.4 for the image of $i_{*}$ unless $p=3$. Thus we have 
Theorem 10.7. $H_{*}\left(\mathscr{M}_{k}, Z / p\right)$ contains elements of the form

$$
z=z\left(I_{1}, \cdots, I_{n}, j_{1}, \cdots, j_{n}\right)=Q^{I_{1}}\left(z_{j_{1}}\right) * \cdots * Q^{I_{n}}\left(z_{j_{n}}\right)
$$

for all sequences $\left(I_{1}, \cdots, I_{n}, j_{1}, \cdots, j_{n}\right)$ such that $\sum_{m=1}^{n} p^{l\left(I_{m}\right)} \leq k$. Here each $I_{m}$ is an admissible sequence with $s_{l}=1, \varepsilon(I)+b(I)>0$ and $j_{a}=0$ or 3 for all $1 \leq a \leq n$. Furthermore the image of $z$ in $H_{*}\left(\mathscr{C}_{k}, Z / p\right)$ is given as follows:

(A) If $p=3$ replace $z_{0}$ by [1] and $z_{3}$ by $-\beta Q^{1}(1) *[-2]$.

(B) If $p \geq 5$ then the image is zero if any $j_{a}=3$. Otherwise replace each $z_{0}$ by $[1]$ thus recovering $10.4(\mathrm{~B})$.

Corollary 10.9. Let $k=3^{j}$. Then

$$
Q^{3^{j}} Q^{3^{j-1}} \cdots Q^{9} Q^{3}\left(z_{3}\right) \in H_{6 k-3}\left(\mathscr{M}_{k}, Z / 3\right)
$$

has nonzero image in $H_{6 k-3}\left(\mathscr{C}_{k}, Z / 3\right)$.

Proof. Just expand $Q^{3^{j}} Q^{3^{j-1}} \cdots Q^{3}\left(-\beta Q^{1}(1) *[-2]\right)$ using the Adem relations and the weight filtration [16].

Corollary 10.10. For $p \geq 5, i_{*}: H_{*}\left(\mathscr{M}_{k}, Z / p\right) \rightarrow H_{*}\left(\mathscr{C}_{k}, Z / p\right)$ has a nontrivial kernel.

Proof. Evaluate $i_{*}\left(z_{3}\right)$ for $0 \neq z_{3} \in H_{3}\left(\mathscr{M}_{1}, Z / p\right)$. q.e.d

Corollary 10.10 is the $\bmod p$ version of Corollary 8.10 for $p \geq 5$. It is tempting to conjecture that the classes $z\left(I_{1}, \cdots, I_{n}, j_{1}, \cdots, j_{n}\right)$ with at least one $j_{a}=3$ also give nontrivial classes in $\operatorname{ker} i_{*}$ for $p \geq 5$. Again we point out this behavior occurs in dimensions $q>k$ and thus is not inconsistent with the Atiyah-Jones conjecture for $q<k$.

Finally we come to $(10.3)$ and the Browder operation $\lambda_{3}(1,1)$, which as we pointed out in $\S 8$ is nonzero and indecomposable in terms of the $Q^{I} \mathrm{~s}$ in $H_{*}\left(\amalg C_{k}\left(R^{4}\right), Z / p\right)$ but zero in $H_{*}\left(\Omega_{2}^{4} B S^{3}, Z / p\right)$. We do not know if $\lambda_{3}(1,1)$ and $Q^{J}\left(\lambda_{3}(1,1)\right)$ are nontrivial in $H_{*}\left(\mathscr{M}_{2}, Z / p\right)$ and $H_{*}\left(\mathscr{M}_{2 p^{l(J)}}, Z / p\right)$; if they are then it is clear that allowing the $z_{j}$ 's in 10.7 to range over [1], $z_{3}$ and $\lambda_{3}(1,1)$ would generate a far larger kernel of $i_{*}$ (again for $q>k$ ).

\section{Some open questions}

We conclude this paper with some comments about future directions that should be pursued.

1. Clearly the main task is to completely determine $H_{*}\left(\mathscr{M}_{k}\right)$ and the image of $i_{*}: H_{*}\left(\mathscr{M}_{k}\right) \rightarrow H_{*}\left(\mathscr{C}_{k}\right)$. The first obvious remark is that while a proof of the Atiyah-Jones conjecture would show $i_{*}$ is an isomorphism through some low dimensional range, 8.10 shows $i_{*}$ has a nontrivial kernel (for $q>k$ ). The following questions arise: 
Is the Browder operation $\lambda_{3}(1,1) \in H_{3}\left(\mathscr{M}_{2}, Z / p\right)$ nontrivial? If so determine the operations $Q^{J}$ for which $Q^{J}\left(\lambda_{3}(1,1)\right)$ is nontrivial. Notice that all these classes belong to the kernel of $i_{*}$.

Is the image of $i_{*}$ completely described by Theorems 9.7 and 10.7? If so $i_{*}$ would fail to be a surjection for $q>k$.

Do the operations given in this paper completely describe $H_{*}\left(\mathscr{M}_{k}\right)$ ? More precisely, do the classes $z\left(I_{1}, \cdots, I_{n}, j_{1}, \cdots, j_{n}\right)$ given in Theorems 9.7 and 10.7 (with perhaps the additional classes obtained by allowing $z_{3}$ to equal $\lambda_{3}(1,1)$ at odd primes) generate $H_{*}\left(\mathscr{M}_{k}, Z / p\right)$ ? Furthermore are there any nontrivial relations between the $z\left(I_{1}, \cdots, I_{n}, j_{1}, \cdots, j_{n}\right)$ 's? Again this question is related to the structure of the kernel of $i_{*}$. For example, are the classes given in 9.10 nontrivial in $H_{*}\left(\mathscr{M}_{2}, Z / p\right)$ ?

2. Is $\mathscr{M}$ an $A_{\infty}$ space? This would imply $\mathscr{M}$ has a classifying space and one could then ask if $\Omega^{3} G$ is a group completion of $\mathscr{M}$. More generally is $(\mathscr{M}, \vartheta)$ a "homotopy $C_{4}$ space" in the sense that all the requisite $C_{4}$ diagrams on $(\mathscr{M}, \vartheta)$ commute up to homotopy? This would imply one could compute Cartan formulas, Adem relations, Nishida relations and so on directly in $H_{*}(\mathscr{M})$ and not just on the image in $H_{*}(\mathscr{C})$.

3. We have given the construction of our structure maps $\vartheta_{j}$, only for the case $G=\operatorname{Sp}(n)$. However both the ADHM construction ( $(4)$ and the Taubes $\varepsilon$ neighborhood theorem (Theorem 2.9) work for $G=\mathrm{SU}(n)$ and $\mathrm{SO}(n)$. Thus one can also construct versions of our structure maps in these cases. To extend the computations of this paper given for $G=\operatorname{Sp}(1)$ to more general groups requires understanding the inclusion $i_{*}: \mathscr{M}_{1} \rightarrow \mathscr{C}_{1}$ in those cases as well as understanding homology operations on $\Omega^{4} \mathrm{Sp}(n), \Omega^{4} \mathrm{SU}(n)$ and $\Omega^{4} \mathrm{SO}(n)$. The Ph.D. thesis of Wagonner [56] should be very valuable in this regard.

4. If the base space $S^{4}$ of the principal bundle $P_{k}$ is replaced by a more general closed, compact, simply connected smooth four-manifold $M$, then do the moduli spaces $\mathscr{M}_{k}$ of self-dual connections still retain the homology operations constructed in this paper? The first manifold to consider is $C P(2)$ where Buchdahl [15] has recently given an ADHM construction for self-dual instantons.

5. Since, by the Ward correspondence, real instanton bundles on $S^{4}$ correspond to certain stable holomorphic vector bundles on $C P(3)$ can our results shed some light on the structure of the moduli space of stable vector bundles on $C P(3)$ ?

6. Atiyah [3], using the results of Donaldson [18], has shown that $\mathscr{M}_{k}$ is diffeomorphic to the space of analytic maps from the Riemann sphere to the analytic loop group $\Omega \mathrm{Sp}(1)$ of degree $k$ where the natural inclusion $i_{k}$ is given by forgetting the analytic structure to obtain maps of degree $k$ from 
$S^{2}$ to $\Omega \mathrm{Sp}(1)$; that is, elements of $\Omega^{3} \mathrm{Sp}(1)$. It would be useful to construct operations in Atiyah's alternate formulation of the instanton problem and identify them with the operations constructed in this paper.

7. We have used the techniques of this paper to study homology operations on moduli spaces of monopoles and certain nonlinear $\sigma$ models [13]. These techniques should also apply to moduli spaces for solutions to other variational problems.

\section{Appendix}

The following tables list the homology classes in $H_{*}\left(\mathscr{M}_{k}, Z / p\right)$ detected by Theorems 9.7 and 10.7 for small values of $k$.

TABLE 1

\begin{tabular}{cc}
$q$ & $H_{q}\left(\mathscr{M}_{1}, Z / 2\right)$ \\
\hline 1 & $z_{1}$ \\
2 & $z_{2}$ \\
3 & $z_{3}$
\end{tabular}

TABLE 2

$$
\frac{q \quad H_{q}\left(\mathscr{M}_{1}, Z / 3\right)}{3}
$$

TABLE 3

\begin{tabular}{|c|c|c|c|}
\hline$q$ & $H_{q}\left(\mathscr{M}_{2}, Z / 2\right)$ & $q$ & $H_{q}\left(\mathscr{M}_{2}, Z / 2\right)$ \\
\hline 1 & $z_{1} *[1]$ & 5 & $Q_{1}\left(z_{2}\right)$ \\
\hline 2 & $z_{1}^{2}$ & 5 & $z_{3} * z_{2}$ \\
\hline 2 & $z_{2} *[1]$ & 5 & $Q_{3}\left(z_{1}\right)$ \\
\hline 3 & $Q_{1}\left(z_{1}\right)$ & 6 & $z_{3}^{2}$ \\
\hline 3 & $z_{2} * z_{1}$ & 6 & $Q_{2}\left(z_{2}\right)$ \\
\hline 3 & $z_{3} *[1]$ & 7 & $Q_{3}\left(z_{2}\right)$ \\
\hline 4 & $Q_{2}\left(z_{1}\right)$ & 7 & $Q_{1}\left(z_{3}\right)$ \\
\hline 4 & $z_{2}^{2}$ & 8 & $Q_{2}\left(z_{3}\right)$ \\
\hline 4 & $z_{3} * z_{1}$ & 9 & $Q_{3}\left(z_{3}\right)$ \\
\hline
\end{tabular}

TABLE 4

\begin{tabular}{cc}
$q$ & $H_{q}\left(\mathscr{M}_{p}, Z / p\right)$ \\
\hline $2 p-3$ & $\beta Q^{1}(1)$ \\
$2 p-2$ & $Q^{1}(1)$
\end{tabular}

The following table catalogs the 19 monomial elements in $H_{q}\left(\mathscr{M}_{4}, Z / 2\right)$ known to exist by Theorem 9.7 that are indecomposable in terms of the loop sum. Using Tables 1 and 3 one can easily construct the other 170 monomial 
elements in $H_{q}\left(\mathscr{M}_{4}, Z / 2\right)$ given by Theorem 9.7 that are loop sum decomposable.

TABLE 5

\begin{tabular}{ll}
$q$ & $H_{q}\left(\mathscr{M}_{4}, Z / 2\right)$ \\
\hline 7 & $Q_{1} Q_{1}\left(z_{1}\right)$ \\
9 & $Q_{3} Q_{1}\left(z_{1}\right)$ \\
9 & $Q_{1} Q_{2}\left(z_{1}\right)$ \\
10 & $Q_{2} Q_{2}\left(z_{1}\right)$ \\
11 & $Q_{1} Q_{1}\left(z_{2}\right)$ \\
11 & $Q_{1} Q_{3}\left(z_{1}\right)$ \\
12 & $Q_{2} Q_{3}\left(z_{1}\right)$ \\
13 & $Q_{3} Q_{3}\left(z_{1}\right)$ \\
13 & $Q_{3} Q_{1}\left(z_{2}\right)$ \\
13 & $Q_{1} Q_{2}\left(z_{2}\right)$ \\
14 & $Q_{2} Q_{2}\left(z_{2}\right)$
\end{tabular}

\begin{tabular}{cc}
$q$ & $H_{q}\left(\mathscr{M}_{4}, Z / 2\right)$ \\
\hline 16 & $Q_{1} Q_{3}\left(z_{2}\right)$ \\
16 & $Q_{1} Q_{1}\left(z_{3}\right)$ \\
17 & $Q_{2} Q_{3}\left(z_{2}\right)$ \\
17 & $Q_{1} Q_{2}\left(z_{3}\right)$ \\
18 & $Q_{2} Q_{2}\left(z_{3}\right)$ \\
18 & $Q_{3} Q_{1}\left(z_{3}\right)$ \\
18 & $Q_{3} Q_{3}\left(z_{2}\right)$ \\
19 & $Q_{1} Q_{3}\left(z_{3}\right)$ \\
20 & $Q_{2} Q_{3}\left(z_{3}\right)$ \\
21 & $Q_{3} Q_{3}\left(z_{3}\right)$
\end{tabular}

TABLE 6

\begin{tabular}{cc}
$q$ & $H_{q}\left(\mathscr{M}_{p^{2}}, Z / p\right)$ \\
\hline $2 p-3$ & $\beta Q^{1}(1)^{*}\left[p^{2}-p\right]$ \\
$2 p-2$ & $Q^{1}(1) *\left[p^{2}-p\right]$ \\
$\vdots$ & $\vdots$ \\
$2(2 p-2)-1$ & $\beta Q^{1}(1) *\left(Q^{1}(1)\right)^{s-1} *\left[p^{2}-s p\right]$ \\
$s(2 p-2)$ & $\left(Q^{1}(1)\right)^{s} *\left[p^{2}-s p\right]$ \\
$\vdots$ & $\vdots$ \\
$p(2 p-2)-1$ & $\beta Q^{1}(1) *\left(Q^{1}(1)\right)^{p-1}$ \\
$p(2 p-2)$ & $Q^{p-1} Q^{1}(1)$ \\
$(p+1)(2 p-3)-1$ & $\beta Q^{p-1} \beta Q^{1}(1)$ \\
$(p+1)(2 p-3)$ & $Q^{p-1} \beta Q^{1}(1)$ \\
$(p+1)(2 p-2)-1$ & $Q^{p} \beta Q^{1}(1)$ \\
$(p+1)(2 p-2)-1$ & $\beta Q^{p} Q^{1}(1)$ \\
$(p+1)(2 p-2)$ & $Q^{p} Q^{1}(1)$
\end{tabular}

for $1 \leq s<p$.

\section{References}

[1] A. Araki \& T. Kudo, Topology of $H_{n}$-spaces and $H$-squaring operations, Mem. Fac. Sci. Kyūshū Univ. Ser. A 10 (1956) 85-120.

[2] M. F. Atiyah, The geometry of Yang-Mills fields, Scuola Norm. Sup. Pisa, 1979. 
[3] __ Instantons in two and four dimensions, Comm. Math. Phys. 93 (1984) 437-451.

[4] M. F. Atiyah \& R. Bott, The Yang-Mills equations over Riemann surfaces, Philos. Trans. Roy. Soc. London Ser. A 308 (1982) 523-615.

[5] M. F. Atiyah, V. G. Drinfeld, N. J. Hitchin \& Y. I. Manin, Construction of instantons, Phys. Lett. A 65 (1978) 185-187.

[6] M. F. Atiyah, N. J. Hitchin \& I. Singer, Self-duality in four dimensional Riemannian geometry, Proc. Roy. Soc. London Ser A 32 (1978) 425-461.

[7] M. F. Atiyah \& J. D. Jones, Topological aspects of Yang-Mills theory, Comm. Math. Phys. 61 (1978) 97-118.

[8] M. F. Atiyah \& R. S. Ward, Instantons and algebraic geometry, Comm. Math. Phys. 55 (1977) 117-124.

[9] W. Barth, Some properties of stable rank-2 vector bundles on $P_{n}$, Math. Ann. 226 (1977) 125-150.

[10] W. Barth \& K. Hulek, Monads and moduli of vector bundles, Manuscripta Math. 25 (1978) 323-347.

[11] J. M. Boardman \& R. M. Vogt, Homotopy everything H-spaces, Bull. Amer. Math. Soc. 79 (1973) 1236-1241.

[12] J. P. Bourguignon \& H. B. Lawson, Yang-Mills theory: Its physical origin and differential geometric aspects, Annals of Math. Studies, No. 102, Princeton University Press, Princeton, NJ, 1982, 395-421.

[13] C. P. Boyer \& B. M. Mann, Monopoles, nonlinear $\sigma$ models and iterated loop spaces, to appear in Comm. Math. Physics.

[14] W. Browder, Homology operations and loop spaces, Illinois J. Math. 4 (1960) 347-357.

[15] N. P. Buchdahl, Instantons on $C P(2)$, J. Differential Geometry 24 (1986) 19-52.

[16] F. R. Cohen, T. J. Lada \& J. P. May, The homology of iterated loop spaces, Lecture Notes in Math., Vol. 533, Springer, Berlin, 1976.

[17] S. K. Donaldson, An application of gauge theory to four dimensional topology, J. Differential Geometry 18 (1983) 279-315.

[18] _ Instantons and geometric invariant theory, Comm. Math. Phys. 93 (1984) 453-460.

[19] __ Anti-self-dual Yang-Mills connections over complex algebraic surfaces and stable vector bundles, Proc. London Math. Soc. 50 (1985) 1-26.

[20] __ Connections, cohomology, and the intersection form of 4-manifolds, J. Differential Geometry 24 (1986) 275-341.

[21] V. G. Drinfeld \& Yu. I. Manin, Self-dual Yang-Mills fields on the sphere, Funktsional Anal. i Prilozhen. 12 (1978) 78-79. (Russian)

[22] __ Locally free sheaves on CP(3) associated to Yang-Mills fields, Uspekhi Mat. Nauk 33 (1978) 241-242. (Russian)

[23] _ Instantons and sheaves on $C P(3)$, Funktsional Anal. i Prilozhen. 13 (1979) 59-74. (Russian)

[24] E. Dyer \& R. K. Lashof, Homology of iterated loop spaces, Amer. J. Math. 84 (1962) 35-88.

[25] R. Fintushel \& R. Stern, SO(3)-connections and the topology of four manifolds, J. Differential Geometry 20 (1984) 523-539.

[26] _ , Pseudofree orbifolds, Ann. of Math. (2) 122 (1985) 335-364.

[27] D. Freed \& K. K. Uhlenbeck, Instantons and four-manifolds, Springer, New York, 1984.

[28] R. Hartshorne, Stable vector bundles and instantons, Comm. Math. Phys. 59 (1978) 1-15.

[29] G. Horrocks, Vector bundles on the punctured spectrum of a local ring, Proc. London Math. Soc. 14 (1964) 684-713.

[30] J. Hurtubise, Instantons and jumping lines, Comm. Math. Phys. 105 (1986) 107-122.

[31] T. Kato, Perturbation theory for linear operators, 2nd ed., Springer, Berlin, 1980. 
[32] S. Kobayashi \& K. Nomizu, Foundations of differential geometry, Vols. I, II, Interscience, New York, 1963, 1969.

[33] H. B. Lawson, The theory of gauge fields in four dimensions, CBMS Regional Conf. Ser. in Math., No. 58, Amer. Math. Soc., Providence, RI, 1985.

[34] B. M. Mann, E. Y. Miller \& H. R. Miller, $S^{1}$-equivariant function spaces and characteristic classes, Trans. Amer. Math. Soc. 295 (1986) 233-256.

[35] J. P. May, A general algebraic approach to Steenrod operations, Lecture Notes in Math., Vol. 168, Springer, Berlin, 1970, 153-231.

[36] _ , The geometry of iterated loop spaces, Lecture Notes in Math., Vol. 271, Springer, Berlin, 1972.

[37] —_, Classifying spaces and fibrations, Mem. Amer. Math. Soc. No. 155, 1975.

[38] R. J. Milgram, Iterated loop spaces, Ann. of Math. (2) 84 (1966) 386-403.

[39] _ The mod-2 spherical characteristic classes, Ann. of Math. (2) 92 (1970) 238-261.

[40] R. L. Mills \& C. N. Yang, Conservation of isotopic spin and isotopic gauge invariance, Phys. Rev. 96 (1954) 191.

[41] S. M. Salamon, Instantons on the 4-sphere, Rend. Sem. Mat. Univ. Politec. Torino 40 (1982) 1-20.

[42] S. Sedlacek, A direct method for minimizing the Yang-Mills functional, Comm. Math. Phys. 86 (1982) 515-528.

[43] G. Segal, Configuration spaces and iterated loop-spaces, Invent. Math. 21 (1973) 213-221.

[44] __ The topology of rational functions, Acta Math. 143 (1979) 39-72.

[45] I. M. Singer, Some remarks on the Gribov ambiguity, Comm. Math. Phys. 60 (1978) 7-12.

[46] J. Stasheff, $H$-spaces from a homotopy point of view, Lecture Notes in Math., Vol. 151, Springer, Berlin, 1970.

[47] N. Steenrod, The topology of fibre bundles, Princeton University Press, Princeton, NJ, 1951.

[48] C. H. Taubes, Self-dual connections on non-self-dual 4-manifolds, J. Differential Geometry 17 (1982) $139-170$.

[49] _ _ Stability in Yang-Mills theories, Comm. Math. Phys. 91 (1983) 235-265.

[50] _ _ Path-connected Yang-Mills moduli spaces, J. Differential Geometry 19 (1984) 337392.

[51] _ Self-dual connections on 4-manifolds with indefinite intersection matrix, J. Differential Geometry 19 (1984) 517-560.

[52] _ , The stable topology of self-dual moduli spaces, preprint, Harvard University, 1986.

[53] G. 't Hooft, On the phase transition towards permanent quark confinement (preprint).

[54] K. K. Uhlenbeck, Connections with $L^{p}$-bounds on curvatures, Comm. Math. Phys. 83 (1982) 31-42.

[55] __ Removable singularities in Yang-Mills fields, Comm. Math. Phys. 83 (1982) 11-30.

[56] D. Waggoner, Loop spaces and the classical unitary groups, Ph. D. Thesis, University of Kentucky, 1985.

[57] G. W. Whitehead, Elements of homotopy theory, Springer, Berlin, 1978. 
